\title{
Precision $\mathrm{Fe} K \alpha$ and $\mathrm{Fe} K \boldsymbol{\beta}$ Line Spectroscopy of the Seyfert 1.9 Galaxy NGC 2992 with Suzaku
}

Tahir Yaqoob, ${ }^{1,2}$ Kendrah D. Murphy, ${ }^{1}$ Richard E. Griffiths, ${ }^{3}$ Yoshito HabA, ${ }^{4}$ Hajime Inoue, ${ }^{5}$ Takeshi IToH, ${ }^{4}$ Richard Kelley, ${ }^{2}$ Motohide Kokubun, ${ }^{6}$ Alex Markowitz, ${ }^{2,7}$ Richard Mushotzky, ${ }^{2}$ Takashi OKajima, ${ }^{1,2}$ Andrew Ptak, ${ }^{1,2}$ James Reeves, ${ }^{1,2}$ Peter J. Serlemitsos, ${ }^{2}$ Tadayuki Takahashi, ${ }^{5}$ and Yuichi Terashima ${ }^{5}$

${ }^{1}$ Department of Physics and Astronomy, Johns Hopkins University, 3400 N. Charles St., Baltimore, MD 21218

${ }^{2}$ Exploration of the Universe Division, NASA Goddard Space Flight Center, Greenbelt Rd., Greenbelt, MD 20771

${ }^{3}$ Department of Physics, Carnegie Mellon University, 5000 Forbes Avenue, Pittsburgh, PA 15213

${ }^{4}$ Department of Astrophysics, School of Science, Nagoya University, Chikusa-ku, Nagoya 464-01, Japan

${ }^{5}$ Institute of Space and Astronautical Science, Japan Aerospace Exploration Agency, 3-1-1 Yoshino-dai, Sagamihara, Kanagawa 229-8510, Japan

${ }^{6}$ Department of Physics, University of Tokyo, 7-3-1 Hongo, Bunkyo-ku, Tokyo, Japan

${ }^{7}$ NASA Postdoctoral Research Associate

(Received DRAFT VERSION 30 July 2006; accepted TBD 2006)

\begin{abstract}
We present detailed time-averaged X-ray spectroscopy in the $0.5-10 \mathrm{keV}$ band of the Seyfert 1.9 galaxy NGC 2992 with the Suzaku X-ray Imaging Spectrometers (XIS). The source had a factor $\sim 3$ higher $2-10 \mathrm{keV}$ flux $\left(\sim 1.2 \times 10^{-11} \mathrm{erg} \mathrm{cm}^{-2} \mathrm{~s}^{-1}\right)$ than the historical minimum and a factor $\sim 7$ less than the historical maximum. The XIS spectrum of NGC 2992 can be described by several components. There is a primary continuum, modeled as a power-law with a photon index of $\Gamma=1.57_{-0.03}^{+0.06}$ that is obscured by a Compton-thin absorber with a column density of $8.0_{-0.5}^{+0.6} \times 10^{21} \mathrm{~cm}^{-2}$. There is another, weaker, unabsorbed power-law component (modeled with the same slope as the primary), that is likely to be due to the primary continuum being electronscattered into our line-of-sight by a region extended on a scale of hundreds of parsecs. We measure the Thomson depth of the scattering zone to be $\tau_{\text {es }}=0.072 \pm 0.021$. An optically-thin thermal continuum emission component, which probably originates in
\end{abstract}


the same extended region, is included in the model and yields a temperature and luminosity of $k T=0.656_{-0.061}^{+0.088} \mathrm{keV}$ and $\sim 1.2 \pm 0.4 \times 10^{40} \mathrm{erg} \mathrm{s}^{-1}$ respectively. We detect an Fe K emission complex which we model with broad and narrow lines and we show that the intensities of the two components are decoupled at a confidence level $>3 \sigma$. The broad $\mathrm{Fe} \mathrm{K} \alpha$ line has an equivalent width of $118_{-61}^{+32} \mathrm{eV}$ and could originate in an accretion disk (with inclination angle greater than $\sim 30^{\circ}$ ) around the putative central black hole. The narrow $\mathrm{Fe} \mathrm{K} \alpha$ line has an equivalent width of $163_{-26}^{+47} \mathrm{eV}$ and is unresolved (FWHM $<4630 \mathrm{~km} \mathrm{~s}^{-1}$ ) and likely originates in distant matter. The absolute flux in the narrow line implies that the column density out of the line-of-sight could be much higher than measured in the line-of-sight, and that the mean (historically-averaged) continuum luminosity responsible for forming the line could be a factor of several higher than that measured from the data. We also detect the $\mathrm{Fe} \mathrm{K} \beta$ line (corresponding to the narrow Fe $\mathrm{K} \alpha$ line) with a high signalto-noise ratio and describe a new robust method to constrain the ionization state of $\mathrm{Fe}$ responsible for the $\mathrm{Fe} \mathrm{K} \alpha$ and $\mathrm{Fe} \mathrm{K} \beta$ lines that does not require any knowledge of possible gravitational and Doppler energy shifts affecting the line energies. For the distant line-emitting matter (e.g. the putative obscuring torus) we deduce that the predominant ionization state is lower than Fe viII (at $99 \%$ confidence), conservatively taking into account residual calibration uncertainties in the XIS energy scale and theoretical and experimental uncertainties in the Fe $\mathrm{K}$ fluorescent line energies. From the limits on a possible Compton-reflection continuum it is likely that the narrow $\mathrm{Fe} \mathrm{K} \alpha$ and $\mathrm{Fe} \mathrm{K} \beta$ lines originate in a Compton-thin structure.

Key words: galaxies: active - galaxies: Seyfert - line:profiles - X-rays:galaxies - X-rays: individual (NGC 2992)

\section{Introduction}

The Fe $\mathrm{K}$ emission line profiles in active galactic nuclei (AGN) generally consist of a prominent core centered at $\sim 6.4 \mathrm{keV}$ (typically with FWHM $<10,000 \mathrm{~km} \mathrm{~s}^{-1}$ or so) and in some sources an additional underlying broader component. The relatively narrow core at $\sim 6.4 \mathrm{keV}$ is found to be ubiquitous in nearby, low to moderate luminosity AGN (e.g. Nandra et al. 1997a,b; Sulentic et al. 1998; Weaver, Gelbord, \& Yaqoob 2001; Reeves 2003; Page et al. 2004; Yaqoob \& Padmanabhan 2004; Jiménez-Bailón et al. 2005; Zhou \& Wang 2005; Jiang, Wang, \& Wang 2006; Levenson et al. 2006). The broad Fe K line component potentially carries signatures of the inner accretion disk in the vicinity of the putative black hole by virtue of gravitational and Doppler energy shifts (e.g. Tanaka et al. 1995; Nandra et al. 1997a,b; Reynolds 1997; Turner et al. 1998; Iwasawa et al. 1999; Yaqoob et al. 2002; Reynolds \& Nowak 2003; Fabian \& Miniutti 2005). The bulk of the Fe $\mathrm{K}$ line core is likely to originate in matter 
located much further from the central black hole than the outer disk (e.g. the outer parts of the optical broad line region, the putative parsec-scale torus structure, or the optical narrow line region). In addition to the two principal components, there are an increasing number of reports of cases of narrow lines at energies higher than $6.4 \mathrm{keV}$ (from ionized and/or outflowing $\mathrm{Fe}$ - see Bianchi et al. 2005, and references therein) and lower than $6.4 \mathrm{keV}$ (from apparently redshifted localized emission - Turner et al. 2002; Iwasawa, Miniutti, \& Fabian 2004; Turner et al. 2006, and references therein).

Deconvolving the disk and distant-matter components of the Fe $\mathrm{K}$ line is extremely challenging because the disk can also contribute to the line core emission (from radii greater than $\sim 100 r_{g} \equiv 100 G M / c^{2}$ or so). In other words, line emission from disks observed at low inclination angles (relative to the disk normal) is partially degenerate with line emission from distant matter. Line emission in the wings of the profile from regions close to the black hole is smeared out over energy and therefore difficult to detect against the continuum. In order to derive the most reliable parameters and constraints for the disk and distant-matter $\mathrm{Fe} \mathrm{K}$ line components, one must simultaneously model both, otherwise the model parameters will be biased and misleading (e.g. see discussions in Weaver \& Reynolds 1998, Yaqoob \& Padmanabhan 2004). So far only some of the brightest AGN have sufficient signal-to-noise ratio to produce useful constraints from dual-line models (e.g. see Yaqoob et al. 2002, Yaqoob \& Padmanabhan 2004, and references therein). For the brightest sources the Fe K line core is usually unresolved even with the Chandra gratings because high spectral resolution and high throughput are required. Therefore, the location of the "distant" Fe K line emitter remains unknown. Nevertheless, decoupling the intensities of the broad and narrow lines (in the sense of showing that the data require both to be non-zero at some confidence level) would be an important step in constraining models of the central engine in AGN. Such decoupling has only been possible for a few of the brightest AGN (e.g. see Fabian \& Miniutti 2005). We also note that detection of the broad Fe $\mathrm{K}$ line in type 1.5-2 AGN is even more difficult than in type 1 objects because the X-ray spectra of the former class are more complex and are usually affected by significant intrinsic X-ray absorption (e.g. see Turner et al. 1998; Weaver \& Reynolds 1998; Matt et al. 2000; Guainazzi et al. 2001; Risaliti 2002).

Almost all of our knowledge of the Fe K line emission in AGN comes from the Fe $\mathrm{K} \alpha$ line. Although Fe $K \beta$ line emission has been reported in a few type 1.5-2 AGN (Sambruna et al. 2001; Molendi, Bianchi, \& Matt 2003; Matt et al. 2004), constraints on the line energy and equivalent width (EW) have been loose because the branching ratio, $\mathrm{Fe} \mathrm{K} \beta / \mathrm{Fe} \mathrm{K} \alpha$, is less than 0.145 (Palmeri et al. 2003, and references therein). Yet the Fe K $\beta$ line carries additional information on the ionization state of line-emitting Fe that would supplement the information carried by the $\mathrm{Fe} \mathrm{K} \alpha$ line.

In this paper we present the results of detailed X-ray CCD spectroscopy of the nearby $(z=0.00771$, Keel 1996) Seyfert 1.9 galaxy NGC 2992 using data from Suzaku observations. 
We show that the intensities of the broad and narrow Fe K $\alpha$ lines are decoupled for the first time in this source. We also detected the $\mathrm{Fe} \mathrm{K} \beta$ line corresponding to the $\mathrm{Fe} \mathrm{K} \alpha$ line core and present measurements of its centroid energy, intensity, and EW. We describe a new method that utilizes the $\mathrm{Fe} K \beta$ line to deduce tight limits on the ionization state of $\mathrm{Fe}$ in the matter responsible for emitting the line core. For the Fe $\mathrm{K} \alpha$ and $\mathrm{Fe} \mathrm{K} \beta$ line analysis we modeled the 0.5-10 keV spectrum self-consistently so we also report the broadband results.

NGC 2992 has been observed by every X-ray astronomy mission since the time it was discovered by HEAO-1 to be one of the brightest hard X-ray AGN in the sky, with a 2-10 keV flux of $\sim 7.2-8.6 \times 10^{-11} \mathrm{erg}^{-2} \mathrm{~s}^{-1}$ (Piccinotti et al. 1982). In more than a quarter of a century of X-ray observations, the hard X-ray flux has varied by over a factor of 20 , corresponding to a range in the intrinsic $2-10 \mathrm{keV}$ luminosity of $\sim 0.55-11.8 \times 10^{42} \mathrm{erg} \mathrm{s}^{-1}$ (assuming $H_{0}=70 \mathrm{~km} \mathrm{~s}^{-1} \mathrm{Mpc}^{-1}, \Lambda=0.73, \Omega=1$ ). During an ASCA observation in 1994, NGC 2992 was in its lowest continuum flux state thus far observed, with a $2-10 \mathrm{keV}$ flux of $\sim 4 \times 10^{-12} \mathrm{erg} \mathrm{cm}^{-2} \mathrm{~s}^{-1}$ (Weaver et al. 1996). During that observation the $\mathrm{Fe} \mathrm{K}$ line equivalent width $(\mathrm{EW})$ was very high $(\sim 500-700 \mathrm{eV})$, indicating that the line intensity had not responded to the declining continuum. In 1997 a BeppoSAX observation found NGC 2992 to be still in a low continuum state, and the $\mathrm{Fe} \mathrm{K}$ line EW was even higher, but then a second BeppoSAX observation in 1998 revealed that the source continuum had fully recovered to its bright HEAO-1 state (see Gilli et al. 2000). In this state, the EW of the Fe $\mathrm{K}$ line was very low $(<100 \mathrm{eV}$ ) since the line intensity again did not respond to the change in continuum level. These measurements were mostly sensitive to the Fe $\mathrm{K}$ line core, which from the lack of variability, likely arises in a distant, parsec-scale reprocessor, as suggested by Weaver et al. (1996).

The results from an XMM-Newton observation of NGC 2992 in May 2003 are unpublished. We examined those data and found that they suffered from heavy pile-up. A detailed analysis of the XMM-Newton data is beyond the scope of this work and will be reported elsewhere.

NGC 2992 was the subject of an RXTE monitoring campaign consisting of 24 observations in the period 2005 March to 2006 January. The Suzaku observations that are the subject of the present paper were made during the $R X T E$ campaign and were quasi-simultaneous with some of the RXTE observations. The scope of the present paper is restricted to the Suzaku data only, but a detailed analysis of the data from the RXTE campaign will be presented elsewhere.

The present paper is organized as follows. In section 2 we describe the observations and details of the data reduction. In section 3 we describe the spectral fitting analysis and in section 4 we discuss the detailed results of that analysis, including brief comparisons with historical data and results. In section 5 we describe a new method to tightly constrain the ionization state of $\mathrm{Fe}$ using the narrow $\mathrm{Fe} \mathrm{K} \alpha$ and $\mathrm{Fe} \mathrm{K} \beta$ emissions lines. In section 6 we summarize our results and give our conclusions. 


\section{Observations and Data Reduction}

The joint Japan/US X-ray astronomy satellite, Suzaku (Mitsuda et al. 2006), was launched on 10 July, 2005. NGC 2992 was observed on three occasions in 2005, November and December (the observation $\log$ is given in table 1). Suzaku carries four X-ray Imaging Spectrometers (XIS - Koyama et al. 2006) and a collimated Hard X-ray Detector (HXD Takahashi et al. 2006). Each XIS consists of four CCD detectors at the focal plane of its own thin-foil X-ray telescope (XRT - Serlemitsos et al. 2006), and has a field-of-view (FOV) of $17.8^{\prime} \times 17.8^{\prime}$. One of the XIS detectors (XIS1) is back-side illuminated (BI) and the other three (XIS0, XIS2, and XIS3) are front-side illuminated (FI). The bandpass of the FI detectors is $\sim 0.4-12 \mathrm{keV}$ and $\sim 0.2-12 \mathrm{keV}$ for the BI detector. The useful bandpass depends on the signal-to-noise ratio of the source since the effective area is significantly diminished at the extreme ends of the operational bandpasses. Although the BI CCD has higher effective area at low energies, the background level across the entire bandpass is higher compared to the FI CCDs. The spectral resolution of the XIS detectors has been continuously degrading with time but the current instrument response matrices employ the resolution before the onset of degradation, as described in Koyama et al. (2006). Although we used these standard response matrices for modeling the XIS data, we measured the widths of the Mn $K \alpha$ lines from the on-board ${ }^{55} \mathrm{Fe}$ calibration sources (two per XIS) using the actual NGC 2992 observations in order to correctly interpret spectral fitting results. Details are given in section 2.3 .

\subsection{HXD Data}

The HXD consists of two non-imaging instruments (the PIN and GSO - see Takahashi et al. 2006) with a combined bandpass of $\sim 10-600 \mathrm{keV}$. Both of the HXD instruments are background-limited. We found that for the NGC 2992 observations the average on-source count rate in the full HXD/PIN band $(\sim 10-120 \mathrm{keV})$ was $\sim 0.75 \mathrm{ct} / \mathrm{s}$, whilst the corresponding estimated background count rate was $0.65 \mathrm{ct} / \mathrm{s}$ so that NGC 2992 constitutes only $\sim 13 \%$ of the total signal. The situation is even worse for the GSO which has a smaller effective area than the PIN. In order to obtain reliable background-subtracted spectra, the background spectrum must be modeled as a function of energy and time. The background model is still under development and cannot yet reproduce the background spectral and temporal behavior with sufficient accuracy to give reliable HXD spectra for NGC 2992. Therefore, in this paper we will restrict our analysis to the XIS only and defer analysis of the simultaneous HXD data to future work when the background model systematics are lower. The observations of NGC 2992 were in fact optimized for the HXD in terms of positioning the source at the aimpoint for the HXD (the so-called "HXD-nominal pointing") which gives a somewhat lower count-rate in the XIS than the "XIS-nominal" pointing but gives 10\% higher HXD effective area. 


\subsection{XIS Data Filtering, Cleaning, and Selection}

The XIS observations of NGC 2992 were made in so-called $3 \times 3$ and $5 \times 5$ edit modes which correspond to 9 and 25 pixel pulse-height arrays respectively that are telemetered to the ground (see Koyama et al. 2006 for details). The array is centered on the pixel containing the largest pulse height of an event. We combined the data from both modes as there did not appear to be any noticeable differences in the spectra obtained from the different modes. Data from version 0.7 of the pipeline reprocessing (Fujimoto et al. 2006; Mitsuda et al. 2006) were used for the analysis in the present paper. As is standard practice, only $A S C A$ grades $0,2,3,4$, and 6 were selected. Event files were cleaned to remove hot and flickering pixels with the FTOOL cleansis as updated for the XIS. Data were excluded during satellite passages through the South Atlantic Anomaly (SAA), and for time intervals less than $256 \mathrm{~s}$ after passages through the SAA, using the T_SAA_HXD house-keeping parameter. Data were also rejected for Earth elevation angles (ELV) less than $5^{\circ}$, Earth day-time elevation angles (DYE_ELV) less than $20^{\circ}$, and values of the magnetic cut-off rigidity (COR) less than $6 \mathrm{GeV} / \mathrm{c}^{2}$. Version 0.7 of the reprocessing pipeline also includes CTI corrections based on available in-flight calibration and other refinements to the XIS energy scale. Residual uncertainties in the energy scale are on the order of $\sim 10 \mathrm{eV}$ (Koyama et al. 2006) and we confirmed this from an analysis of the onboard calibration line data taken during the NGC 2992 observations (see section 2.3 for details).

The cleaning and data selection resulted in net exposure times in the range $31.6 \mathrm{ks}$ to $41.7 \mathrm{ks}$ for the three observations (see table 1). We examined XIS images made from the cleaned events files and found that the galaxy NGC 2993 ( $\sim 3^{\prime}$ from the core of NGC 2992) was clearly detected but its total count rate was less than $1 \%$ of the NGC 2992 count rate. Even though the half-power diameter of the XRT+XIS is 2', NGC 2992 and NGC 2993 are resolved. NGC 2993 was detected by Chandra with much better spatial resolution (see Colbert et al. 2005). We extracted XIS lightcurves and spectra using two sets of circular regions with radii of 2.6' and 4.35' centered on NGC 2992. The smaller region excludes NGC 2993, resulting in negligible contamination of the NGC 2992 spectra, and the larger region corresponds to the size assumed by some of the standard XRT effective area files that have been released. The spectra from the smaller regions will be used for the principal analysis and those from the larger regions will be used to better estimate absolute fluxes (see below). We extracted background spectra using circular regions with radii of $\sim 4^{\prime}$ from source-free regions of the detectors.

\subsection{Instrument Response Files and the Mean XIS Spectrum}

For spectral analysis we used the response matrices (or "RMF" files) ae_xi0_20060213.rmf, ae_xi1_20060213c.rmf, ae_xi2_20060213.rmf, and ae_xi3_20060213.rmf for XIS0, XIS1, XIS2 and XIS3 respectively (binned to the same number of channels as the spectra). The telescope effective area files (or "ARF" files) used were ae_xi0_hxdnom4_20060415.arf, ae_xi1_hxdnom4_20060415.arf, 
ae_xi2_hxdnom4_20060415.arf and ae_xi3_hxdnom4_20060415.arf for XIS0, XIS1, XIS2, and XIS3 respectively. The ARF files are standard files released for the HXD-nominal pointing position and assume a circular extraction with a radius of $2.9^{\prime}$. Since our source extraction regions had a radius of $2.6^{\prime}$ we derived a mean correction factor by comparing broadband fluxes measured from the spectra extracted using 4.35' radii regions with those from our 2.6' radius region. The ARF files used for the spectra from the larger regions were ae_xi0_hxdnom6_20060415.arf, ae_xi1_hxdnom6_20060415.arf, ae_xi2_hxdnom6_20060415.arf and ae_xi3_hxdnom6_20060415.arf for XIS0, XIS1, XIS2, and XIS3 respectively. Although the larger regions include NGC 2993, the contamination is small and the procedure is adequate for the present purpose.

Some preliminary examination and spectral fitting revealed no significant evidence for variability in the total count rate or spectral shape within each of the three observations. Although there was up to $\sim 30 \%$ variability in net count rate between observations, we found no statistically significant evidence for spectral variability between the observations (see table 1). We found that the four XIS sensors were consistent with each other to $\sim 10 \%$ or better (within statistical errors) over the energy band 0.5-10 keV. However, XISO showed a systematic dip in the $\sim 5-7 \mathrm{keV}$ band (i.e. in the region that $\mathrm{Fe} \mathrm{K} \alpha$ line emission is expected) which was not apparent in the other three XIS sensors. Also, XIS1 showed a broad dip in the $\sim 2-5 \mathrm{keV}$ band which was not apparent in the other three XIS sensors. Although both of these effects are at the level of $\sim 10 \%$ or less, for the sake of maximum conservatism, in the remainder of this paper all of the spectral analysis will refer to a single XIS spectrum combined from observations 1,2 and 3 , using only XIS2 and XIS3 data. For analysis of the mean spectrum from XIS2+XIS3 (integrated over the three observations), we combined the RMF and ARF files (four in total) into one response file with appropriate weightings, according to the exposure times of the data segments. The mean $0.5-10 \mathrm{keV}$ background-subtracted count rate for this spectrum was $0.4442 \pm 0.0016 \mathrm{ct} / \mathrm{s}$ per XIS, and the net exposure time was $1.08 \times 10^{5} \mathrm{~s}$ per XIS. We restricted the analysis to the $0.5-10 \mathrm{keV}$ band in order to avoid background-subtraction systematics (in the $0.5-10 \mathrm{keV}$ band the background constitutes $\sim 3.4 \%$ of the total count rate). We obtained a flux correction factor for the size of the extraction region of $2.1 \%$ (as described above) for the mean spectrum. All fluxes and absolute line intensities quoted in this paper have been corrected (increased) by this factor.

We measured the centroid energies of the $\mathrm{Mn} K \alpha$ lines from the calibration sources, using a background-subtracted spectrum combined from XIS2 and XIS3 data, summed over observations 1-3 (i.e. the same combination used for the analysis of NGC 2992), and summed over both calibration sources on each XIS. The expected energies of the $\mathrm{Mn} K \alpha_{1}$ and $\mathrm{Mn} K \alpha_{2}$ lines are $5.89875 \mathrm{keV}$ and $5.88765 \mathrm{keV}$ respectively (Bearden 1967). Since the $K \alpha_{1}: K \alpha_{2}$ branching ratio is $2: 1$, the expected centroid energy is then $5.89505 \mathrm{keV}$. Using a single Gaussian we measured a centroid energy of $5.906 \pm 0.003 \mathrm{keV}$ (one-parameter, $90 \%$ confidence error). 
Therefore, the difference between the measured and expected centroid energy is $11 \pm 3 \mathrm{eV}$, consistent with Koyama et al. (2006). Both the residual CTI correction uncertainty and the spectral resolution are better at the centers of the detectors (i.e. in the region of the aimpoints for source observations) than at the detector edges where the calibration sources are located so this is an over-estimate. We also measured the Mn $K \alpha$ line width and obtained a FWHM of $161.5 \mathrm{eV}$ (at an energy of $\sim 5.9 \mathrm{keV}$ ). At $6.4 \mathrm{keV}$ this corresponds to a FWHM of $168.4 \mathrm{eV}$ (see Koyama et al. 2006), or $\sim 7890 \mathrm{~km} \mathrm{~s}^{-1}$ FWHM. Since the FWHM resolution at $6.4 \mathrm{keV}$ assumed in the response matrices is $133.8 \mathrm{eV}$ (from direct inspection), there is a residual FWHM width of $34.6 \mathrm{eV}$ (or $1620 \mathrm{~km} \mathrm{~s}^{-1}$ at $6.4 \mathrm{keV}$ ) that is not currently accounted for in the instrument response function. Again, the spectral resolution is better at the center of the detectors (by $\sim 5-10 \mathrm{eV}$ ) than at the edges.

\subsection{XIS Contaminant Correction}

The XIS detectors have suffered a loss in low-energy efficiency due to absorption by a contaminant on the optical blocking filter. The degradation can be modeled using "effective" column densities of Carbon and Oxygen that can be calculated as a function of time since launch and position on each XIS, based on empirical fits to in-flight data obtained from non-varying astrophysical sources (see Koyama et al. 2006). We calculated the Carbon column density for each XIS and each observation of NGC 2992. For analysis of the mean spectrum described above we then calculated the average of the six column densities for XIS2 and XIS3, observations 1 to 3 , and obtained $3.4 \times 10^{18} \mathrm{~cm}^{-2}$. Based on the current assumption that the contaminant is probably $\mathrm{C}_{24} \mathrm{H}_{38} \mathrm{O}_{4}$, the $\mathrm{O}$ column density was fixed at $1 / 6$ of the value of the $\mathrm{C}$ column density. The $\mathrm{C}$ and $\mathrm{O}$ column densities were then combined with photoelectric absorption cross-sections from Balucinska-Church \& McCammon (1992) since these were used to derive the empirical degradation formulae. The resulting contamination model, implemented using the varabs model in XSPEC, was included along with all astrophysical models of NGC 2992 (see section 3).

\section{Spectral Fitting}

In the remainder of this paper we shall refer to analysis of the combined spectrum from XIS2 and XIS3 averaged over all three observations (table 1), as described in section 2.3. We used XSPEC v11.3.2 for spectral fitting (Arnaud 1996), utilizing data in the $0.5-10 \mathrm{keV}$ band. The instrument response files used were described in section 2.3. The spectrum was binned to $\sim 29 \mathrm{eV}$ per bin and this resulted in $>25 \mathrm{ct} /$ bin for the net spectrum over the $0.5-9.7 \mathrm{keV}$ range so we were able to use the $\chi^{2}$ statistic for finding the best-fitting model and for the statistical error analysis. Unless otherwise stated, all statistical errors given correspond to $90 \%$ confidence for one interesting parameter $\left(\Delta \chi^{2}=2.706\right)$. Galactic absorption with a column density of $5.26 \times 10^{20} \mathrm{~cm}^{-2}$ (Dickey \& Lockman 1990) was included in all of the models 
described hereafter and its inclusion will be implicitly assumed. For the Galactic absorption, as well as any intrinsic absorption, we used photoelectric cross-sections given by Morrison \& McCammon (1983). The correction for the XIS contamination degradation (as described in section 2.4) was also included in all of the spectral fitting described below. All astrophysical model parameter values will be given in the rest frame of NGC 2992.

\subsection{Preliminary Spectral Fitting and the Baseline Model}

Preliminary spectral fitting confirmed the complexity in the X-ray spectrum of NGC 2992 that has been established from observations with previous X-ray astronomy missions (see section 1). This complexity is illustrated in figure 1(a), which shows the ratio of the XIS data to a simple model consisting of a power-law intrinsic continuum and uniform intrinsic absorption fully covering the source. There are only three free parameters in this model, namely the normalization of the power-law continuum, its photon index, $\Gamma$, and the column density, $N_{H}$. For this model $\chi^{2}=1158.2$ for 321 degrees of freedom. Relative to this simple model there is a large excess at soft X-ray energies below $\sim 2 \mathrm{keV}$ and a very prominent Fe K $\alpha$ emission line. In addition, a weaker emission line is apparent at $\sim 7 \mathrm{keV}$, which could be due to Fe XxVI Ly $\alpha$, Fe $\mathrm{K} \beta$, or a blend of both. From a detailed analysis described in section 5 we identify the weaker line with Fe $K \beta$ and show quantitatively that contamination by any Fe xxvi Ly $\alpha$ line emission is negligible.

At least part of the soft excess in figure 1(a) is due to scattering of the intrinsic X-ray continuum into the line-of-sight by an extended optically-thin zone in the active nucleus (e.g. Turner et al. 1998). This scattered component has been imaged in NGC 2992 using ROSAT and Chandra (see Colbert et al. 2005, and references therein) and in some other Seyfert 1.5-2 galaxies (e.g. Morse et al. 1995; Ogle et al. 2000; Young, Wilson, \& Shopbell 2001; George et al. 2002; Iwasawa et al. 2003). The direct continuum along the line-of-sight is obscured so even if only a small fraction of the intrinsic continuum is scattered, the soft X-ray spectrum can easily be dominated by the scattered component. Therefore, in the next step we added another power-law continuum component to the model, with the photon index tied to that of the direct continuum and a normalization that was allowed to float. We parameterized this normalization in terms of the fraction, $f_{s}$, of the directly observed power-law continuum normalization. In the optically-thin limit the Thomson depth, $\tau_{\mathrm{es}}$, of the scattering region (assuming spherical symmetry) is simply equal to $f_{s}$. We also added two unresolved Gaussian components to model the emission lines in the Fe $\mathrm{K}$ band. The intrinsic widths $\left(\sigma_{N}\right)$ of the two lines were tied together (in this case the two emission lines originate from the same atoms/ions). In the preliminary spectral fitting $\sigma_{N}$ was fixed at a value $(0.005 \mathrm{keV})$ much less than the XIS spectral resolution. The other parameters were the centroid energies of the two emission lines $\left(E_{\mathrm{Fe} K \alpha}\right.$ and $\left.E_{\mathrm{Fe} \mathrm{K} \beta}\right)$, and their intensities ( $I_{\mathrm{Fe} K \alpha}$ and $I_{\mathrm{Fe} K \beta}$ respectively). Thus, in this more complex model there were a total of eight free parameters $\left(\Gamma, N_{H}, f_{s}, E_{\mathrm{Fe} K \alpha}, E_{\mathrm{Fe} K \beta}, I_{\mathrm{Fe} \mathrm{K} \alpha}, I_{\mathrm{Fe} \mathrm{K} \beta}\right.$, and a continuum 
normalization). The best-fitting model gave $\chi^{2}=400.6$ for 316 degrees of freedom. The ratio of the XIS data to this best-fitting model is shown in figure 1(b). The fit is still poor and statistically significant residuals are still evident in the soft X-ray band below $\sim 2 \mathrm{keV}$ and in the Fe $\mathrm{K}$ band. Note that if we included only one Gaussian component (to model the strongest emission line) we obtained $\chi^{2}=418.8$ for 318 degrees of freedom, demonstrating that the weaker emission line is statistically significant (see section 5 for a more quantitative analysis). We then allowed the intrinsic width $\left(\sigma_{N}\right)$ of the Gaussian emission-line components to be free and refitted the model, obtaining another significant reduction in $\chi^{2}$, which dropped to 375.4 for 315 degrees of freedom.

Motivated by the fact that an extended soft thermal emission component has been detected in NGC 2992 (see Colbert et al. 2005) and in other type 1.5-2 Seyfert galaxies (e.g. Ptak et al. 1997; Bianchi, Guainazzi, \& Chiaberge 2006, and references therein), we then added a component to model optically-thin thermal emission. We used the APEC model in XSPEC with the element abundances fixed at cosmic values (but later we allowed the Fe abundance to be free in order to derive constraints on it). The APEC model then involves two more free parameters, $k T$, and $L_{\mathrm{APEC}}$, the temperature and luminosity of the plasma respectively (although we used the normalization of the APEC model as the actual free parameter in the fits, not its luminosity). In all, there were now eleven free parameters and we obtained $\chi^{2}=354.1$ for 313 degrees of freedom.

There still remained some residuals in the Fe $\mathrm{K}$ band indicative of additional, broader, line emission. This might be expected from an X-ray illuminated accretion disk whose inner radius extends to within $\sim 10$ or so gravitational radii of the putative central black hole. Such relativistically broadened Fe $\mathrm{K}$ line emission has been observed in other AGN (e.g. Fabian \& Miniutti 2005, and references therein). Indeed, it is possible that the Gaussian emission-line components in the above preliminary model could be modeling part of the disk line emission as well as the narrow core emission that might originate from more distant matter. With CCD data the line core can be degenerate with part of the disk line component because both components can have significant emission near the line rest-frame energy. Even though the Fe $\mathrm{K}$ line core may be the dominant component, it is in general necessary to simultaneously model both the broad and narrow components otherwise the inferred model parameters for one component will be biased by the presence of the unmodeled component. For example, in the spectral fits described above with the Gaussian widths free, the best-fitting lines may be broader than the true width of the Fe $\mathrm{K}$ line core because they may be trying to model an underlying broad component as well.

Therefore, we added Fe K line emission from a relativistic disk around a (Schwarzschild) black hole using the diskline model in XSPEC (see Fabian et al. 1989). The diskline model has six parameters, namely the energy of the line in the disk frame, $E_{0}$; the inner and outer radii of the disk ( $R_{\mathrm{in}}$ and $R_{\text {out }}$ respectively, in units of gravitational radii, $r_{g} \equiv G M / c^{2}$ ); the 
power-law index, $q$, of the radial line emissivity law $\left(r^{q}\right)$; the inclination angle of the disk normal relative to the observer's line-of-sight, $\theta_{\text {obs }}$; and the integrated intensity of the line, $I_{\text {disk }}$. The overall Fe $\mathrm{K}$ line profile in the XIS data is already dominated by the line core and we found that the data for any residual broad-line emission could not constrain all of the disk line model parameters. In particular, there is considerable degeneracy between $E_{0}$ and $\theta_{\text {obs }}$. Therefore, we fixed $E_{0}$ at $6.40 \mathrm{keV}$ (corresponding to the rest-frame energy of $\mathrm{Fe}$ I $\mathrm{K} \alpha$ ). In addition, we fixed $R_{\text {in }}$ and $R_{\text {out }}$ at $6 r_{g}$ (the radius of the last stable orbit around a Schwarzschild black hole) and $1000 r_{g}$ respectively. For self-consistency we also included a disk line component corresponding to Fe I K $\beta$ with no additional free parameters: $R_{\text {in }}, R_{\text {out }}, q$, and $\theta_{\text {obs }}$ were forced to take the same values as the $\mathrm{Fe} \mathrm{K} \alpha$ line, $E_{0}$ was fixed at $7.056 \mathrm{keV}$, and the intensity of the $\mathrm{Fe} \mathrm{K} \beta$ line was forced to be $13.5 \%$ of the $\mathrm{Fe} \mathrm{K} \alpha$ line intensity (see section 5 for a detailed discussion on Fe $\mathrm{K} \beta$ line energies and the $\mathrm{Fe} \mathrm{K} \beta / \mathrm{K} \alpha$ branching ratio). Although there may be no clear isolated detection of the broad $\mathrm{Fe} \mathrm{K} \beta$ line, for ionization states of $\mathrm{Fe} \mathrm{I}$ to $\mathrm{Fe}$ XVI it will be produced, and excluding it from the model could affect the values of the inferred parameters of the other Fe $\mathrm{K}$ line components. Fitting the XIS data with the Fe $\mathrm{K}$ disk line emission components included in the model results in a drop in $\chi^{2}$ of 19.7 (to 334.4 for 310 degrees of freedom) for an extra three free parameters $\left(q, \theta_{\text {obs }}\right.$, and $I_{\text {disk }}$ ), indicating that a broad-line component is required by the data with a confidence level of more than $3 \sigma$.

Finally, we added a component to model a Compton-reflection continuum using the model href 1 in XSPEC (see Dovčiak, Karas, \& Yaqoob 2004). Although only the $\sim 7-10 \mathrm{keV}$ band XIS data are able to constrain the reflection continuum, if any of the $\mathrm{Fe} \mathrm{K}$ emission line components originate in Compton-thick matter, a reflection continuum will be produced. Excluding a reflection continuum in the model could bias the best-fitting parameters of the Fe $\mathrm{K}$ emission lines. The reflection model involves only one extra free parameter, $R$, which is the normalization of the reflection continuum relative to the case of a steady-state $X$-ray continuum illuminating a slab subtending a solid angle of $2 \pi$ at the $X$-ray source. The inclination angle between the disk normal and the observer's line-of-sight was tied to the disk inclination angle for the relativistic Fe $\mathrm{K}$ emission-line components. Note that a disk geometry is likely to be inappropriate for any reflection continuum associated with Compton-thick distant-matter components of the $\mathrm{Fe} \mathrm{K}$ line emission, but the data are not sensitive to differences in the reflection continuum due to geometry. When we fitted the above model to the data we found a best-fitting value of $R=0$, and consequently no improvement in the fit. Nevertheless, we retained the reflection continuum in all of the spectral fitting analysis (with $R$ free), since it could potentially affect the statistical error analysis for the other parameters.

Thus, our baseline model consisted of fifteen free parameters: two normalizations (one for the soft, optically-thin thermal continuum, another for the intrinsic power-law continuum), $k T, \Gamma, N_{H}, f_{s}, \sigma_{N}, E_{\mathrm{Fe} \mathrm{K} \alpha}, I_{\mathrm{Fe} \mathrm{K} \alpha}, E_{\mathrm{Fe} \mathrm{K} \beta}, I_{\mathrm{Fe} \mathrm{K} \beta}, q, \theta_{\mathrm{obs}}, I_{\text {disk }}$, and $R$. This model gave an excellent fit to the data ( $\chi^{2}=334.4$ for 309 degrees of freedom), as can be seen from figure 2 (a) 
(which shows the best-fitting model overlaid on the XIS counts spectrum), figure 2 (b) (which shows the best-fitting model and some of the individual model components), and figure 2 (c) (which shows the ratio of the XIS data to the best-fitting model). The best-fitting parameters and $90 \%$, one-parameter statistical errors are shown in table 2 . We discuss these results in detail in section 4 .

\subsection{Deconvolution of the Broad and Narrow Fe $K$ Emission Lines}

Figure 3(a) shows a close-up of the Fe K region of the XIS spectrum of NGC 2992 in the form of the ratio of the data to a model consisting of a simple power-law continuum with a fully-covering absorber (i.e. figure 3(a) is simply a "zoomed' region from figure 1(a)). Note that the $\mathrm{Fe} \mathrm{K} \beta$ line is clearly seen here. A broad base to the prominent $\mathrm{Fe} \mathrm{K} \alpha$ line core is also apparent. In section 3.1 we found that when we modeled the underlying broad emission with a disk line, the Gaussian components that modeled the line core emission became narrower than for the case when the broad line emission was not modeled. In fact the best-fitting baseline model (table 2) indicates that the line cores of $\mathrm{Fe} \mathrm{K} \alpha$ and $\mathrm{Fe} \mathrm{K} \beta$ are unresolved by the XIS data. In order to better illustrate the $\mathrm{Fe} \mathrm{K}$ line profile due to the putative relativistic disk emission, we removed the disk line model components from the baseline model, froze the parameters of the thermal emission, reflection continuum, and Gaussian line core models and re-fitted the data. The resulting ratio of data to model is shown in figure 3(b) which illustrates the profile of the relativistic disk component of the Fe $\mathrm{K}$ line.

Next, starting from the baseline model again, with its fifteen free parameters (section 3.1) we constructed joint confidence contours of the equivalent width (EW) of the $\mathrm{Fe} \mathrm{K} \alpha$ line core versus its intrinsic width (FWHM). The 68\%, 90\%, 99\% confidence contours are shown in figure $3(\mathrm{c})$ which demonstrates that the $99 \%$, two-parameter upper limit on the FWHM appears to be $\sim 6300 \mathrm{~km} \mathrm{~s}^{-1}$. However, as mentioned in section 2 the response matrices do not include the time-dependent degradation in the XIS energy resolution. In section 2.3 we showed that analysis of the Mn $K \alpha$ calibration line data taken during the NGC 2992 observations gave a residual FWHM width of $34.6 \mathrm{eV}$ (or $1620 \mathrm{~km} \mathrm{~s}^{-1}$ at $6.4 \mathrm{keV}$ ) that is not accounted for by the current response matrices. Since the observed line width from any observed source is a convolution of the intrinsic line width and the instrumental broadening, the true $99 \%$ confidence, two-parameter upper limit of the Fe Ko line core in NGC 2992 is then $\left[6300^{2}-1620^{2}\right]^{\frac{1}{2}} \sim 6090 \mathrm{~km} \mathrm{~s}^{-1}$. Note that the narrow-line widths and their one-parameter statistical errors in table 2 have also been corrected for this effect but the contours in figure 3(c) are not corrected. The relativistic disk line parameters have not been corrected. The disk line is then observed to be a little broader than it really is so that our lower limit on the disk inclination angle is slightly over-estimated and the radial emissivity parameter, $q$, should be slightly smaller.

We also constructed joint confidence contours of the intensity of the Fe $\mathrm{K} \alpha$ disk line 
versus the $\mathrm{Fe} \mathrm{K} \alpha$ intensity of the narrow, distant-matter line. During this process, as for figure $3(\mathrm{c})$, all of the thirteen remaining parameters in the baseline model were free. The resulting contours are shown in figure $3(\mathrm{~d})$, for $68 \%, 90 \%, 99 \%$, and $3 \sigma$ respectively. Remarkably, it can be seen that at a confidence level of greater than $3 \sigma$, the disk and distant-matter $\mathrm{Fe} \mathrm{K} \alpha$ lines are decoupled in the sense that the intensity of either of the two line components is non-zero everywhere on the $3 \sigma$ contour.

We investigated the possibility that part of the disk line may be an artifact due to the current XIS response matrices inadequately modeling the low-energy tail of the response function (see Koyama et al. 2006). Again we used the Mn $K \alpha$ calibration line backgroundsubtracted spectrum, integrated over observations 1-3, summed over XIS2 and XIS3, using both calibration sources on each XIS. We modeled the main peak with a disk line and Gaussian line, plus a simple power-law continuum. The disk-line model parameters (see section 3.1) $E_{0}$, $R_{\text {in }}, R_{\text {out }}$ were fixed at the values used for modeling NGC 2992 and the values of $\theta_{\text {obs }}$, and $q$ were fixed at the best-fitting values shown in table 2. The disk line and Gaussian line intensities, as well as the peak energy and width of the Gaussian component were free parameters. By constructing confidence contours of the disk line intensity versus the Gaussian line intensity we established that the low-energy excess in the actual in-flight XIS response can only account for a disk line intensity that is $2 \%$ of the Gaussian line intensity, with a $99 \%$ confidence twoparameter upper limit of $\sim 6 \%$. Yet for the actual NGC 2992 data we obtained a best-fitting $\mathrm{Fe} \mathrm{K} \alpha$ disk line intensity that was $76 \%$ of the line core (see table 2). Even the $3 \sigma$ confidence contour of the disk line intensity versus the line core intensity (see figure 3(d)) only goes down to a value of $\sim 8 \%$ for the ratio of the disk line to line core intensity. Therefore the low-energy excess in the tail of the XIS response does not have a significant impact on the measurements of the disk line in the NGC 2992 data or its decoupling from the Fe $\mathrm{K} \alpha$ line core.

Decoupling of the Fe K $\alpha$ components from the accretion disk and distant matter has not been possible with previous data for NGC 2992 (see section 4.4). It is even more remarkable that the decoupling has been possible with CCD resolution data and for a relativistic disk line that is not particularly impressive in terms of signal-to-noise ratio (figure 3(b)) or EW (see table 2). The reason can be traced to the fact that the narrow line has excellent signal-to-noise and a large EW. This in turn leaves less freedom for the disk line model. While it is possible that there may not be two distinct physical locations of $\mathrm{Fe} \mathrm{K} \alpha$ line emission, a single line-emitting region would demand a peculiar line-emissivity law as a function of radius. The strength of the line core compared to the broad base (which we model as a disk line) would demand that the emissivity fall as a function of radius and then increase again. The width of the broad base of the Fe K $\alpha$ line (see figure 3(b)) is $\sim 33,000 \mathrm{~km} \mathrm{~s}^{-1} \mathrm{FWHM}$, more than five times the upper limit on the width of the unresolved line core (figure 3(c)). Yet a uniform spherical distribution of matter gives line profiles which have much less contrast in the line width between the center and wings of the profile (e.g. see Yaqoob et al. 1993). The implications of the parameters derived 
from the dual Fe $\mathrm{K} \alpha$ line modeling will be discussed further in section 4.4 and compared with published results on historical data.

\section{Discussion of Results}

In this section we discuss in detail the results that were obtained by fitting the baseline model (section 3.1) to the XIS data (see table 2).

\subsection{Soft, Optically-thin Thermal Emission}

We obtained a temperature and luminosity of the optically-thin thermal emission component of $k T=0.656_{-0.061}^{+0.088} \mathrm{keV}$ and $\sim 1.2 \pm 0.4 \times 10^{40} \mathrm{erg} \mathrm{s}^{-1}$ respectively. The temperature is typical of that found for a similar component in other type 1.5-2 AGN (e.g. see Ptak et al. 1997; Bianchi et al. 2006, and references therein). Note that the errors quoted here on the temperature are statistical only and do not include systematic errors, which may be larger. Chandra was able to image the soft X-ray emission better than Suzaku but was still not able to spatially separate it from other emission components due to contamination from the PSF wings caused by the dominant nuclear emission (Colbert et al. 2005). Compounded with this were CCD pileup issues, and lower signal-to-noise ratio of the data. Therefore Chandra obtained somewhat worse constraints on the temperature of the soft thermal emission. Colbert et al. (2005) give $k T=0.51_{-0.19}^{+0.26} \mathrm{keV}$ and $L=1.4 \times 10^{40} \mathrm{erg} \mathrm{s}^{-1}$ and conclude that the bulk of the soft X-ray emission originates from within $\sim 150-500 \mathrm{pc}$ of the central engine. The temperature and luminosity of the extended soft emission component obtained by Chandra are both consistent with our respective values measured with Suzaku.

Our Suzaku measurement of the temperature of the soft thermal emission in NGC 2992 is the most reliable to date. Elvis et al. (1990) reported detecting the soft X-ray emission component in NGC 2992 using the Einstein HRI, but it was only detected in one out of four quadrants, the temperature was poorly constrained, and the luminosity was more than an order of magnitude greater than that obtained by Suzaku and Chandra. Although the soft $\mathrm{X}$-ray emission imaged by the ROSAT HRI was spatially consistent with Chandra (see Colbert et al. 2005), the ROSATHRI could not measure the temperature since it was not a spectroscopic instrument. The ROSAT PSPC spectral resolution was too poor to measure the temperature. $A S C A$ CCD spectra had better spectral resolution but the signal-to-noise ratio of the NGC 2992 $A S C A$ data is lower than that of the Suzaku data (see Weaver et al. 1996). BeppoSAX could not constrain the soft thermal component because both the MECS and LECS instruments had poorer spectral resolution than the Suzaku XIS and the useful MECS bandpass did not extend to below $\sim 0.9 \mathrm{keV}$. 


\subsection{Intrinsic Continuum and Absorption}

We obtained $\Gamma=1.57_{-0.03}^{+0.06}$ for the power-law photon index of the intrinsic continuum (see table 2). This is somewhat less than that obtained from BeppoSAX ( $\Gamma \sim 1.7$ - Gilli et al. 2000) and Chandra ( $\Gamma \sim 1.8$ - Colbert et al. 2005) observations. ASCA could not constrain $\Gamma$ for the complex models required to explain the data (Weaver et al. 1996). Given the model-dependence of $\Gamma$ and residual instrument cross-calibration uncertainties of the order of $\Delta \Gamma \sim 0.1$, we do not interpret the apparent differences in $\Gamma$ amongst the different observations as necessarily having an astrophysical origin.

We derived a column density of $8.0_{-0.5}^{+0.6} \times 10^{21} \mathrm{~cm}^{-2}$ for the line-of-sight X-ray obscuration (see table 2) which corresponds to a Thomson depth of $\sim 0.006_{-0.004}^{+0.005}$ (i.e. the absorber is Compton-thin, at least in the line-of-sight). This column density is consistent with values obtained from previous observations with BeppoSAX (Gilli et al. 2000) and ASCA (Weaver et al. 1996).

We tested the data to see if any additional $\mathrm{Fe} \mathrm{K}$ absorption was required by adding a simple absorption-edge model (zedge in XSPEC) with the threshold energy fixed at $7.11 \mathrm{keV}$ (corresponding to neutral $\mathrm{Fe}$ ) but allowing the optical depth at the threshold energy to be a free parameter. All the other 15 free parameters in the model remained free. We obtained a best-fitting threshold optical depth of zero and a one-parameter, $90 \%$ confidence upper limit of 0.06 . Since the optical depth at the Fe K edge threshold energy for the absorption already included in the model is $\sim 0.008$ (for $N_{H}=8 \times 10^{21} \mathrm{~cm}^{-2}$ and the Fe abundance used), we could only obtain a loose upper limit on the possible over-abundance of Fe of $\sim 7.5$.

Table 2 gives the observed XIS fluxes (including the effect of Galactic and intrinsic absorption but not the CCD contaminant) in the $0.5-2 \mathrm{keV}$ and 2-10 keV bands which were 0.16 and $1.15 \times 10^{-11} \mathrm{erg} \mathrm{cm}^{-2} \mathrm{~s}^{-1}$ respectively. However, note that systematic uncertainties in the absolute flux calibration of X-ray detectors, as indicated by flux reference values quoted for the Crab Nebula observed by different instruments, are generally a few percent but can be as high as 10-20\% in some cases (e.g. see Jahoda et al. 2006, and references therein). Historically, the lowest and highest 2-10 keV observed fluxes for NGC 2992 have been measured by $A S C A$ in $1994\left(\sim 0.4 \times 10^{-11} \mathrm{erg} \mathrm{cm}^{-2} \mathrm{~s}^{-1}\right.$ - Weaver et al. 1996), and by HEAO-1 in 1978 $\left(\sim 8.6 \times 10^{-11} \mathrm{erg} \mathrm{cm}^{-2} \mathrm{~s}^{-1}-\right.$ see Piccinotti et al. 1982) respectively. This represents a factor $\sim 21.5$ in dynamic range. The Suzaku flux is at the lower end of the historically observed range, but it is nearly a factor $\sim 3$ higher than the historical minimum.

Table 2 also gives the inferred XIS luminosities after correcting for absorption. These were calculated by setting the Galactic and intrinsic absorbing column densities to zero in the best-fitting baseline model (section 3.1). We obtained 0.7 and $1.6 \times 10^{42} \mathrm{erg} \mathrm{s}^{-1}$ in the $0.5-2 \mathrm{keV}$ and $2-10 \mathrm{keV}$ (rest-frame) bands respectively (assuming $H_{0}=70 \mathrm{~km} \mathrm{~s}^{-1} \mathrm{Mpc}, \Lambda=0.73, \Omega=1$ ). The historical range in 2-10 keV luminosity is therefore $\sim 0.55-11.8 \times 10^{42} \mathrm{erg} \mathrm{s}^{-1}$. 


\subsection{Scattered Continuum}

Table 2 shows that we measured $(7.2 \pm 2.1) \%$ for the continuum scattered into the line-ofsight (as a percentage of the direct line-of-sight continuum before absorption). This translates directly into $\tau_{\mathrm{es}}=0.072 \pm 0.021$ for the extended, warm, electron scattering zone. The scattered fraction and optical depth cannot be easily compared with results from previous observations. The $A S C A$ data had very poor signal-to-noise and the model in Weaver et al. 1996 did not include extended thermal emission. The BeppoSAX data did not have coverage of the soft X-ray band with sufficient resolution to measure $f_{s}$ (see Gilli et al. 2000).

Colbert et al. (2005) determined from Chandra ACIS data that the scattering zone must be situated at more than $\sim 150 \mathrm{pc}$ from the nucleus since the spectrum from inside this region could be fitted with a power-law and absorption only. However, despite Chandra having much better spatial resolution than Suzaku, the Chandra data could not constrain the optical depth of the electron-scattering zone because the scattered continuum is spatially confused with the soft thermal emission and even with some of the direct central emission, due to the PSF wings. Spectroscopically, the Chandra ACIS data are inferior to the Suzaku data because they suffer from severe pile-up and have a much lower signal-to-noise ratio than the Suzaku data. For example, the $0.3-8 \mathrm{keV}$ nuclear, and "near-nuclear" Chandra spectra analyzed by Colbert et al. (2005) had $\sim 6,744$ and $\sim 14,600$ counts respectively, as opposed to a total of nearly $\sim 10^{5}$ counts $(0.5-10 \mathrm{keV})$ in the XIS spectrum that we have analyzed here. Note also that pile-up in the Chandra data prohibited a reliable measurement of the flux and luminosity of the nuclear source. Thus, even though the spatial resolution of Suzaku is not as good as Chandra, the high signal-to-noise ratio in the Suzaku spectrum and absence of pile-up problems allowed a better measurement of the scattered component and hence a better estimate of the Thomson depth of the scattering zone.

\subsection{Fe K Emission Lines}

In section 3.2 and figure 3(d) we showed that the intensities of the narrow and broad $\mathrm{Fe}$ K $\alpha$ emission-line components were decoupled. The best-fitting line parameters and statistical errors are shown in table 2. We obtained only a lower limit on the disk inclination angle, $\theta_{\mathrm{obs}}>31^{\circ}$. The actual value is somewhat dependent on the model used for the broad line (here it is the simple Schwarzschild black hole disk model which does not include light bending - see Fabian et al. 1989). However, the overall roughly symmetric shape of the line (figure 3(b)) is a characteristic that excludes small inclination angles, independent of the detailed model, since smaller angles would give rise to an increasing asymmetry on the red side due to gravitational redshifting. We obtained an $\mathrm{EW}$ of $118_{-61}^{+32} \mathrm{eV}$ for the broad line, which is consistent with that expected from a "cold" accretion disk illuminated by the observed direct X-ray continuum (e.g. see George \& Fabian 1991). The index of the line emissivity radial power law, $q=-1.5_{-0.8}^{+5.1}$, is flatter than the average value for Seyfert 1 galaxies (e.g. Nandra et al. 1997a) but it is sensitive 
to the inner and outer disk radius parameters, which we held fixed for the model-fitting (see section 3.1). We tried fixing $q$ at the steeper value of -3 and obtained a lower limit on $\theta_{\text {obs }}$ and $R_{\text {in }}$ of $29^{\circ}$ and $31 r_{g}$ respectively.

For the centroid energy of the Fe $\mathrm{K} \alpha$ line core (which we modeled with a Gaussian component), we obtained $6.407 \pm 0.007 \mathrm{keV}$ (table 2). The statistical errors on this centroid energy are comparable to the residual systematic errors in the absolute energy scale of the XIS (on the order of $\sim 10 \mathrm{eV}$ or so - see section 2.3, Koyama et al. 2006). For the corresponding Fe K $\beta$ line we obtained a centroid energy of $7.034_{-0.038}^{+0.037} \mathrm{keV}$. The $68 \%, 90 \%$, and $99 \%$ confidence contours of the narrow $\mathrm{Fe} K \beta$ line energy versus the $\mathrm{Fe} K \alpha$ line energy are shown in figure $4(\mathrm{a})$, and figure 4(b) shows corresponding contours for the line intensities of Fe $\mathrm{K} \alpha$ and Fe $\mathrm{K} \beta$. Note that both sets of contours were constructed from the baseline model (section 3.1) allowing, in each case, all thirteen of the remaining parameters to be free. The measured energies and statistical errors on the $\mathrm{Fe} \mathrm{K} \alpha$ and $\mathrm{Fe} \mathrm{K} \beta$ line energies are a function of calibration uncertainties, Doppler and gravitational shifts, and the ionization state of Fe. Further analysis utilizing information from the $\mathrm{Fe} \mathrm{K} \beta$ line, including the $\mathrm{Fe} \mathrm{K} \beta / \mathrm{K} \alpha$ ratio, is described in section 5 .

The measured intensity of the narrow (distant-matter) Fe K $\alpha$ line, $I_{N}=2.5_{-0.4}^{+0.7} \times$ $10^{-5}$ photons $\mathrm{cm}^{-2} \mathrm{~s}^{-1}$ (see table 2 ), is a factor of $\sim 20$ times larger than the line intensity expected from a fully-covering Compton-thin spherical shell with the cosmic abundance of Fe used in this paper, the measured line-of-sight column density $\left(8.0_{-0.5}^{+0.6} \times 10^{21} \mathrm{~cm}^{-2}\right)$, and the measured intrinsic 2-10 keV luminosity (see table 2). Using the expressions in Yaqoob et al. 2001 (see also Krolik \& Kallman 1987), we obtained a predicted intensity of $1.1 \times 10^{-6}$ photons $\mathrm{cm}^{-2} \mathrm{~s}^{-1}$. Therefore, if the line emitter is Compton-thin, one or more of the following must be true: (i) the $\mathrm{Fe}$ abundance is higher than the assumed cosmic value, (ii) the column density out of the line-of-sight is greater than that measured along the line-of-sight, and (iii) there are time delays between changes in the amplitude of the X-ray continuum luminosity and the response of the narrow $\mathrm{Fe} \mathrm{K} \alpha$ line so that the observed line intensity may correspond to a time-averaged continuum illumination. On the other hand, the EW of $163_{-26}^{+47} \mathrm{eV}$ measured for the narrow Fe $\mathrm{K} \alpha$ line is also compatible with that expected from a line observed from the X-ray illuminated inner surface of a Compton-thick torus-like structure (although the historically averaged illuminating luminosity may need to be higher than the luminosity directly observed, e.g. see Makishima 1986; Ghisellini, Haardt, \& Matt 1994). However, if the $\mathrm{Fe} \mathrm{K \alpha}$ line core were produced in Compton-thick distant matter we would expect to observe the so-called "Compton shoulder" on the red side of the line core. The Compton shoulder would have manifested itself as an asymmetry in the $E_{\mathrm{Fe} \mathrm{K} \beta}$ versus $E_{\mathrm{Fe} K \alpha}$ contours shown in figure $4(\mathrm{a})$, in the sense that they would be more elongated towards the lower energies (e.g. see Yaqoob et al. 2005 and references therein). Also, upper limits on the expected Compton-reflection continuum appear to be too low (section 4.5). Thus, the distant-matter $\mathrm{Fe} K \alpha$ line is likely to originate in Compton-thin matter. 
Table 2 and figure 3(c) show that the Fe $\mathrm{K} \alpha$ line core (narrow component) is unresolved by the XIS, and we obtained a $90 \%$ confidence, one-parameter upper limit of $4630 \mathrm{~km} \mathrm{~s}^{-1}$ on the FWHM (or $\sigma_{N}<42 \mathrm{eV}$ ). These upper limits on the line width have been corrected (as described in section 3.2) for the fact that the current instrument response matrices do not model the time-dependent degradation of the XIS spectral resolution (see Koyama et al. 2006). The measured upper limits on the line width can be compared with a theoretical estimate of FWHM $\sim 760 \sqrt{\left(M_{8} / r_{\mathrm{pc}}\right)} \mathrm{km} \mathrm{s}^{-1}$ (assuming a virial relation and an r.m.s. velocity dispersion of $\sqrt{3} V_{\text {FWHM }} / 2$; e.g. see Netzer 1990). Here, $M_{8}$ is the central black-hole mass in units of $10^{8} M_{\odot}$, and $r_{\mathrm{pc}}$ is the distance (in parsecs), of the line-emitting structure from the central mass. The mass of the central black hole in NGC 2992 has been estimated to be $5.2 \times 10^{7} M_{\odot}$ from stellar velocity dispersions (Woo \& Urry 2002, and references therein). Therefore, the measured upper limit on the FWHM corresponds to $r_{\mathrm{pc}}>0.034$, or $r>40.2$ light days. For comparison, broad $\mathrm{Pa} \beta$ line emission has been observed in NGC 2992 with a FWHM of $\sim 1200 \mathrm{~km} \mathrm{~s}^{-1}$ (Rix et al. 1990) indicating $\sim 250$ light days for the location of the putative hidden BLR (see also Veilleux, Goodrich, \& Hill 1997).

It is pertinent to ask whether the narrow $\mathrm{Fe} \mathrm{K} \alpha$ line core has varied in intensity compared to historical data (see Weaver et al. 1996; Gilli et al. 2000, and references therein). Given the large historical amplitude of variability of the continuum luminosity (greater than a factor of 20 ), the response of the narrow $\mathrm{Fe} \mathrm{K} \alpha$ line to the continuum would give some clue about the size of the line-emitting structure. In order to answer the line variability question one needs to fit spectra with broad and narrow $\mathrm{Fe} \mathrm{K} \alpha$ line models simultaneously, but this has not been done in the literature. We have fitted historical ASCA and BeppoSAX data with the dual Fe $\mathrm{K}$ line model used for the Suzaku data and have found that at the $99 \%$ confidence level, the ASCA (1994) and BeppoSAX (1997) data are consistent with no variability of the narrow Fe K $\alpha$ line, and the BeppoSAX (1998) data cannot rule out variability up to a factor of $\sim 5$. This is a factor of $\sim 4$ less than the continuum variability and supports an origin of the narrow Fe K $\alpha$ line core in distant matter. At $99 \%$ confidence, the intensity of the broad (disk) Fe K $\alpha$ line during the low-state $A S C A$ and BeppoSAX (1997) observations is consistent with no variability compared to the Suzaku measurement, but the high state BeppoSAX (1998) data cannot rule out a factor of up to $\sim 15$ variability. Therefore the relation between the broad line intensity and continuum luminosity remains open to future investigation. We note that in the 1997 BeppoSAX data (when NGC 2992 was in a near-minimum flux state), there were additional residuals at $\sim 6.8 \mathrm{keV}$ (rest-frame) relative to the dual-line model possibly due to emission from Fe XXV and/or Fe XXVI (see discussion in Gilli et al. 2000).

\subsection{Compton-Reflection Continuum}

If the broad $\mathrm{Fe} \mathrm{K}$ line originates in an X-ray illuminated accretion disk then basic physics predicts a specific Compton-reflection continuum shape and amplitude commensurate with the 
Fe K line parameters (e.g. George \& Fabian 1991; Magdziarz \& Zdziarski 1995) and the disk ionization state. We used a simple disk reflection model (see section 3.1) to parameterize the total reflection continuum from all possible locations (e.g. the accretion disk and any Comptonthick distant matter) because only a small portion of the XIS bandpass $(\sim 7-10 \mathrm{keV})$ can possibly constrain it. The best-fitting value of the so-called reflection fraction, $R$, was $0 . R$ is the normalization of the reflection continuum relative to that expected from a steady-state X-ray illuminated neutral disk subtending a solid angle of $2 \pi$ at the X-ray source. Although the XIS data cannot constrain the reflection continuum very well, we included it in the model because omitting it could potentially affect the inferred values of some of the other model parameters, especially those of the broad disk line. We found that the upper limits on $R$ were strongly dependent on the disk inclination angle, principally because of the angle-dependence of the relativistic line profile shape (the angular dependence of the reflection continuum shape in the restricted energy band is negligible). Therefore, we constructed joint $68 \%, 90 \%$, and $99 \%$ confidence contours of $R$ versus $\theta_{\text {obs }}$, and these are shown in figure 5 . It can be seen that the $90 \%$ confidence, two-parameter upper limit on $R$ varies from $\sim 0.6$ to 0.9 as $\theta_{\text {obs }}$ varies from its lower limit of $\sim 30^{\circ}$ to $\sim 80^{\circ}$ respectively (the spectral fit is not well-behaved as we approach $\sim 90^{\circ}$ ). For comparison with values in the literature, the $90 \%$, one-parameter upper limits on $R$ are 0.60 and 0.78 for fixed inclination angles of $\theta_{\mathrm{obs}}=43^{\circ}$ (the best-fitting value) and $\theta_{\mathrm{obs}}=60^{\circ}$ respectively (see also table 2 ).

We checked that the limits on the Compton-reflection continuum were not sensitive to background subtraction systematics by constructing a completely new background spectrum from rectangular regions from off-source locations in XIS2 and XIS3 and summed over the three observations. Note that the nominal background spectrum used thus far was constructed from circular regions centered at different positions and as far as possible relative to the new background regions (see section 2.2). With the new background spectrum in place, the baseline model with fifteen free parameters was re-fitted and there was no statistically distinguishable change in any of the parameters. The one-parameter, $90 \%$ confidence upper limit on $R$ for $\theta_{\text {obs }}=43^{\circ}$ remained at 0.60 and the corresponding upper limit for $\theta_{\text {obs }}=60^{\circ}$ decreased from 0.78 to 0.76 . Note in figure 5 how the data strongly reject models with small inclination angles $\left(\theta_{\text {obs }}<20^{\circ}\right.$ is ruled out at $>99 \%$ confidence). This is further confirmation that the narrow and broad Fe $\mathrm{K}$ lines are decoupled because in poorer quality data a low-inclination angle disk line model can fit the narrow core of the Fe $\mathrm{K}$ line profile even if the line core has a non-disk origin.

We also checked that the disk line intensity was not sensitive to the amplitude of the reflection continuum in the fit by constructing confidence contours of the disk line intensity, $I_{\text {disk }}$, versus $R$ (and reverting to the nominal background spectrum for consistency with the earlier analysis). We found that that the $99 \%$ confidence lower limit on $I_{\text {disk }}$ was fairly flat and non-zero over the range $0<R<3$, and was never less than $0.4 \times 10^{-5}$ photons $\mathrm{cm}^{-2} \mathrm{~s}^{-1}$.

Under the stated assumptions of our model we would expect $R=1$. The one-parameter 
upper limits on $R$ discussed above are not necessarily inconsistent with the measured EW $\left(118_{-61}^{+32} \mathrm{eV}\right)$ of the broad Fe $\mathrm{K}$ line. The small bandpass leverage may bias $R$, and our model for the reflection continuum does not include relativistic smearing (which would tend to lower the effective value of $R$ ). The model also assumes a cold, neutral disk with a cosmic Fe abundance. However, our upper limits on $R$ do imply that there is unlikely to be a significant Compton-reflection continuum from the structure that produces the core of the Fe $\mathrm{K}$ fluorescent line emission (e.g. the putative obscuring torus), which in turn implies that the structure is Compton-thin.

Unfortunately the PDS data from both of the BeppoSAX observations, including the high-state observation in 1998, were too noisy to obtain useful measurements or limits on a Compton-reflection continuum (see Gilli et al. 2000). Although Colbert et al. (2005) claimed to measure a Compton-reflection continuum in the off-nuclear region of NGC 2992 using Chandra, the data only extended up to $8 \mathrm{keV}$, the signal-to-noise was poor, and the data suffered from severe pile-up. The models fitted were complex in relation to the quality of the data and the uniqueness of the deconvolution of the different spectral components under such circumstances is an issue that was not addressed. In the future we expect the background modeling and subtraction systematics for the HXD onboard Suzaku to improve and we will revisit the challenge of measuring the Compton-reflection continuum in NGC 2992. Nevertheless, it is important to note that the detection of the relativistic disk line in the XIS data is insensitive to uncertainties in the reflection continuum: there was no preferred model solution in which a non-zero value of $R$ forced the relativistic disk line intensity to zero.

\subsection{Upper Limits on the Ni/Fe Abundance}

We investigated Ni $K \alpha$ line emission in the XIS data by adding another Gaussian line component to the baseline model (section 3.1). The intrinsic width of the additional Gaussian was tied to that of the Fe $\mathrm{K} \alpha$ and $\mathrm{Fe} \mathrm{K} \beta$ lines. Since visual inspection revealed little evidence for Ni $K \alpha$ line emission we fixed the center energy of the Gaussian at the expected (rest-frame) energy of $7.472 \mathrm{keV}$ (Bearden 1967). Thus, only the intensity of the $\mathrm{Ni} K \alpha$ line was a free parameter, giving a total of sixteen free parameters in the model. The results are shown in table 2 , and it can be seen that a statistically significant detection was not obtained. The decrease in the fit statistic was only $\Delta \chi^{2} \sim 1.1$. We obtained a $90 \%$ confidence, one-parameter upper limit on the EW of $28 \mathrm{eV}$.

If the Fe K $\alpha$ and Ni $K \alpha$ lines are formed in Compton-thin matter, the ratio of their intensities depends principally on the respective ratios of the fluorescence yields, the $K \beta / K \alpha$ branching ratios, the photoelectric absorption cross-sections, and the $\mathrm{Fe}$ and $\mathrm{Ni}$ abundances. Using Bambynek et al. (1972) for the fluorescence yields and $K \beta / K \alpha$ branching ratios, and Henke ${ }^{1}$ for photoelectric absorption cross-sections, we evaluated equation (3) in Yaqoob et al. (2001) for

1 ww-cxro.1bl.gov 
Fe and $\mathrm{Ni}$ and obtained the theoretical ratio of line intensities: $I_{\mathrm{Ni} \mathrm{K} \alpha} / I_{\mathrm{Fe} \mathrm{K} \alpha}=0.96\left(A_{N i} / A_{F e}\right)$. From the XIS data we constructed a joint $90 \%$ confidence contour of $I_{\mathrm{Ni} \alpha}$ versus $I_{\mathrm{Fe} \mathrm{K} \alpha}$ and obtained an upper limit on $I_{\mathrm{Ni} \mathrm{K} \alpha} / I_{\mathrm{Fe} \mathrm{K} \alpha}$ of 0.176 . Thus we obtained a $90 \%$ confidence upper limit on the $\mathrm{Ni} / \mathrm{Fe}$ abundance ratio of 0.183 . For comparison, the $\mathrm{Ni} / \mathrm{Fe}$ abundance ratio from the Anders \& Grevesse (1989) solar values is 0.038 (i.e. a factor 4.8 smaller than our upper limit). If the $\mathrm{K} \alpha$ lines were observed in reflection from Compton-thick matter then the expected $I_{\mathrm{Ni} \alpha} / I_{\mathrm{Fe} \mathrm{K} \alpha}$ ratio would be less than the Compton-thin case (due to absorption of the $\mathrm{Ni} K \alpha$ by $\mathrm{Fe}$ ) and our upper limit on the $\mathrm{Ni} / \mathrm{Fe}$ abundance would be larger.

\section{Precision X-ray Spectroscopy with the Fe K $\alpha$ and Fe K $\beta$ Lines}

In this section we will constrain the ionization state of $\mathrm{Fe}$ responsible for the narrow $\mathrm{Fe} \mathrm{K}$ line emission in a robust manner by using as much information as possible from the spectrum and by utilizing the latest atomic physics data and associated uncertainties. Firstly, we can make an immediate deduction from the very fact that the $\mathrm{Fe} \mathrm{K} \beta$ fluorescence line is detected at all. That is, Fe must be less ionized than Fe XVII in the line-emitting matter responsible for the narrow Fe K line emission because Fe XVII and ions with higher ionization states have no $M$ shell electrons. We can constrain the ionization state further by taking advantage of the fact that we measured the $\mathrm{Fe} \mathrm{K} \alpha$ and $\mathrm{Fe} \mathrm{K} \beta$ line energies with sufficiently small statistical errors. We cannot do this directly from the measured line energies (e.g. using figure 4(a)) because they could be affected by residual XIS energy scale uncertainties, possible gravitational redshifts, and possible Doppler shifts. We will describe a method which enables us to place an upper limit on the dominant ionization state of $\mathrm{Fe}$ that is independent of the residual uncertainty in XIS gain, and does not require any knowledge of the possible gravitational and Doppler shifts. Our result will depend only on the uncertainty in the absolute offset in the XIS energy scale, on which we can confidently place robust limits in general (Koyama et al. 2006), and for for every observation using the onboard calibration sources. However, we first justify our confidence that the $\mathrm{Fe} \mathrm{K} \beta$ line suffers from negligible contamination from possible $\mathrm{Fe} \mathrm{K} \alpha$ line emission.

In figure $4(\mathrm{a})$ we showed the $68 \%, 90 \%$, and $99 \%$ confidence contours of $E_{\mathrm{Fe} \mathrm{K} \beta}$ versus $E_{\mathrm{Fe} \text { Ka }}$ measured from the XIS data. The fact that the contours are fairly symmetric about the best-fit and are not elongated towards lower Fe $\mathrm{K} \beta$ energies implies that there is little or no blending from Fe XXVI Ly $\alpha$, whose rest-frame centroid energy would be at $6.966 \mathrm{keV}$ (e.g. Pike et al. 1996). We added another Gaussian line component to the baseline model (section 3.1) at a fixed energy of $6.966 \mathrm{keV}$, with its intrinsic width tied to that of the $\mathrm{Fe} \mathrm{K} \alpha$ and $\mathrm{Fe} \mathrm{K} \beta$ lines and obtained a best-fit Fe XXVI Ly $\alpha$ EW of 0 and a $90 \%$, one-parameter upper limit of $18.5 \mathrm{eV}$.

The $68 \%, 90 \%$, and $99 \%$ confidence contours of $I_{\mathrm{Fe}} \mathrm{K} \beta$ versus $I_{\mathrm{Fe} \mathrm{K} \alpha}$ measured from the XIS data are shown in figure 4(b). The lower and upper dashed lines correspond to $I_{\mathrm{Fe}} \mathrm{K}_{\beta} / I_{\mathrm{Fe} \mathrm{K} \alpha}$ ratios of 0.12 and 0.135 respectively. Palmeri et al. (2003) give a detailed discussion of the theo- 
retical and experimental values of the $\mathrm{Fe} \mathrm{K} \beta / \mathrm{Fe} \mathrm{K} \alpha$ branching ratio. They compare theoretical values of the ratio in the literature for Fe I to Fe IX calculated using different codes and assumptions and they compare these theoretical values with several experimental measurements for Fe I. Palmeri et al. (2003) show that for Fe I, except for one experimental value, the remainder of the theoretical and experimental values of the $\mathrm{Fe} \mathrm{K} \beta / \mathrm{Fe} \mathrm{K} \alpha$ branching ratio lie in the range $\sim 0.12-0.135$. Including the highest experimental value, the range is $\sim 0.12-0.145$ corresponding to a dispersion of $\sim 20 \%$. For higher ionization states of Fe the branching ratio increases, and Palmeri et al. (2003) show that the theoretical value for Fe IX can be as high as $\sim 0.17$. The actual value of the observed ratio of the $\mathrm{Fe} \mathrm{K} \beta$ to $\mathrm{Fe} \mathrm{K} \alpha$ line depends a little on the optical depth of the medium in which the lines originate since the two lines have a slightly different albedo.

New calculations for the energies of fluorescent Fe K $\alpha$ and Fe K $\beta$ transitions, fluorescent yields, and rates, for all charge states of Fe have been presented by Palmeri et al. (2003), Mendoza et al. (2004), Bautista et al. (2003), and Kallman et al. (2004). These works also include a comparison with previous calculations (and measurements, where appropriate) in the literature. In figure 4(a), for Fe I-Fe IX, we have plotted the expected Fe $\mathrm{K} \beta$ line energies against the expected Fe $\mathrm{K} \alpha$ line energies (solid, filled circles), overlaid on the measured Fe $\mathrm{K} \beta$ line energy versus Fe $K \alpha$ line energy confidence contours. The energies were calculated from Palmeri et al. (2003), weighted according to the appropriate branching ratios for $K \alpha_{1}$ and $K \alpha_{2}$, and for $K \beta_{1}$ and $K \beta_{2}$ where necessary. As discussed in detail by Kallman et al. (2004), and references therein, for ionization states higher than Fe IX the number of transitions increases, and a single line centroid energy for comparison with observations becomes less and less meaningful since the fluorescent line profile shape then depends on the details of the excitation mechanisms in play. One must then compute the detailed photoionization equilibrium in order to derive theoretical line profiles to compare with observations. From a comparison of results of theoretical calculations with laboratory measurements (solid state for Fe I, EBIT results for Fe IX), Kallman et al. (2004) assess the systematic error in the Fe K fluorescent line energies to be $\sim 0.5 \mathrm{~m} \AA$ for Fe I and $\sim 2 \mathrm{~m} \AA$ for Fe IX. We note that in $\mathrm{eV}$, a systematic error of $\Delta \lambda$ in $\mathrm{m} \AA$, is $\Delta E(\mathrm{eV}) \sim 3.3 \Delta \lambda$ and $\Delta E(\mathrm{eV}) \sim 4.0 \Delta \lambda$ at $6.4 \mathrm{keV}$ and $7.0 \mathrm{keV}$ respectively.

It is important to note from the filled circles in figure $4(\mathrm{a})$ that although the theoretical Fe K $\beta$ line energy increases along the sequence Fe I to Fe IX, the Fe $\mathrm{K} \alpha$ line energy does not increase for these ionization states. This was pointed out by Palmeri et al. (2003) and Kallman et al. (2004) and the results are essentially a refinement of the results of Makishima (1986) which are universally quoted to describe a monotonic increase in the $\mathrm{Fe} \mathrm{K} \alpha$ line energy with increasing charge state of Fe. However, Makishima (1986) never gave numerical values for the Fe $\mathrm{K} \alpha$ line energies, and for the ionization states Fe I to Fe IX it is not possible to extract the line energy values from their plot with sufficient precision to compare with the values from Palmeri et al. (2003). Although the decrease in the Fe K $\alpha$ line energy in going from Fe I to Fe IX 
is less than $10 \mathrm{eV}$, the XIS data for NGC 2992 are sensitive to these small energy differences. The statistical and systematic errors on the Fe Ko line energy measured from the NGC 2992 XIS data are comparable to the difference in the theoretical values of the Fe I and Fe IX line energies. The values of the $\mathrm{Fe} \mathrm{K} \alpha$ and $\mathrm{Fe} \mathrm{K} \beta$ line energies, the associated statistical errors, and confidence contours measured for NGC 2992 are not only a function of the predominant charge state of Fe and residual calibration uncertainties, but are also affected by possible Doppler and gravitational shifts. In order to make inferences on the ionization state of Fe from the XIS data we must consider all of these factors, including uncertainties in the theoretical line energies, so we cannot simply deduce the ionization states allowed by the data from figure $4(a)$.

In order to constrain the dominant ionization stage of Fe further, we can recast the $E_{\mathrm{Fe} \mathrm{K} \beta}$ versus $E_{\mathrm{Fe} \mathrm{K} \alpha}$ contours shown in figure 4(a). For a given ionization stage of Fe there are a pair of "true" (or theoretical) values of $E_{\mathrm{Fe} \mathrm{K} \alpha}$ and $E_{\mathrm{Fe} K \beta}$. Suppose that the observed and true values of $E_{\mathrm{Fe} \alpha}$ and $E_{\mathrm{Fe} K \beta}$ are linearly related by

$$
E_{i ; \mathrm{Fe} \mathrm{K} \alpha} \text { (observed) }=A+B \times E_{i, \mathrm{Fe} \mathrm{K} \alpha}(\text { true })
$$

and

$$
E_{i, \mathrm{Fe} \mathrm{K} \beta} \text { (observed) }=A+B \times E_{i, \mathrm{Fe} \mathrm{K} \beta} \text { (true), }
$$

where $i=0$ to 25 represents the ionization state of Fe (i.e. the number of electrons missing in the ion relative to the neutral atom), $A$ represents any residual calibration uncertainties in the offset of the absolute energy scale and $B$ includes contributions from residual uncertainties in the XIS gain as well as possible gravitational and/or Doppler shifts. Certainly, over the restricted energy range being considered here, any higher order terms in the gain uncertainty are negligible (see Koyama et al. 2006), and the gravitational and Doppler terms only make a linear contribution. Therefore, the linear equations above are an excellent representation of the relations between the true rest-frame line energies and the final line energies measured by the XIS.

Since $A$ and $B$ are the same for the Fe K $\alpha$ and Fe K $\beta$ lines, we can solve for $A$ and $B$ for a given ionization stage of $\mathrm{Fe}$ for any given pair of measurements of $E_{i, \mathrm{Fe}} \mathrm{K} \alpha$ (observed) and $E_{i, \mathrm{Fe} \mathrm{K} \beta}$ (observed). Thus we can recast a contour of the measured energies $E_{\mathrm{Fe} \text { K } \beta}$ and $E_{\mathrm{Fe} \mathrm{K} \alpha}$ for a given confidence level into a contour of $B$ versus $A$ for every $i$ (i.e. we can generate up to 25 contours from figure $4(\mathrm{a})$ ). Since we have independent information on the calibration of the absolute energy scale of the XIS data, we can then rule out any ionization states that yield $B$ versus $A$ contours that give solutions for $A$ that are inconsistent with the known accuracy of the XIS energy scale.

The $99 \%$ confidence contours of $B$ versus $A$ calculated as described above are shown in figure 6 for $\mathrm{Fe}$ I to $\mathrm{Fe}$ IX, using the expected $\mathrm{Fe} \mathrm{K} \alpha$ and $\mathrm{Fe} \mathrm{K} \beta$ line energies calculated from Palmeri et al. (2003). These energies were also shown as filled circles in figure 4(a). The vertical dashed lines in figure 6 represent the uncertainty in the absolute energy scale offset of the XIS 
(i.e. they enclose the possible range of values of $A$ ). Although we have stated that this is nominally $\sim \pm 10 \mathrm{eV}$, we will base our conclusions on the very conservative assumption that $|A|$ is less than $50 \mathrm{eV}$. The vertical dashed lines are therefore at $A= \pm 50 \mathrm{eV}$. It can be seen from figure 6 that the contours for Fe VII to Fe IX are ruled out regardless of the value of B. We can go further and take into account the uncertainties in the theoretical values of the Fe $\mathrm{K} \alpha$ and $\mathrm{Fe} \mathrm{K} \beta$ line energies. The $B$ versus $A$ contours depend on $E_{\mathrm{Fe} \mathrm{K} \beta}-E_{\mathrm{Fe} \mathrm{K} \alpha}$ and we considered the two extreme cases, corresponding to the theoretical difference in these energies hypothetically being in error by $-2 \mathrm{~m} \AA$ and $+2 \mathrm{~m} \AA$. We recalculated the $B$ versus $A$ confidence contours for each of these cases for Fe I to Fe IX. The cases for a theoretical error of $-2 \mathrm{~m} \AA$ and $+2 \mathrm{~m} \AA$ gave contours which ruled out ionization states higher than Fe V and Fe vII respectively. Therefore, we can say that at $99 \%$ confidence the narrow core of the Fe K $\alpha$ line in NGC 2992 originates in matter in which the predominant ionization state of Fe lies in the range Fe I-Fe viI. We emphasize that this conclusion requires no knowledge of the uncertainty in the XIS gain, or of any possible gravitational or Doppler shifts experienced by the photons leaving the source.

\section{Summary and Conclusions}

We have presented detailed X-ray spectroscopy in the $0.5-10 \mathrm{keV}$ band of the Seyfert 1.9 galaxy NGC 2992 with the Suzaku XIS. Here we summarize the results.

1. The mean observed $2-10 \mathrm{keV}$ flux of NGC 2992 during the three Suzaku observations in 2005 November and December was $\sim 1.2 \times 10^{-11} \mathrm{erg} \mathrm{cm}^{-2} \mathrm{~s}^{-1}$, a factor of $\sim 3$ higher than the historic minimum flux, and a factor of $\sim 6$ lower than the historic maximum. The absorption-corrected intrinsic 2-10 keV luminosity was $1.6 \times 10^{42} \mathrm{erg} \mathrm{s}^{-1}$.

2. We detected the extended, optically-thin soft X-ray thermal emission found with previous $\mathrm{X}$-ray astronomy missions, and obtained a temperature of $k T=0.656_{-0.061}^{+0.088} \mathrm{keV}$. This is the most precise measurement of $k T$ for NGC 2992 to date although the errors are purely statistical and do not include any possible systematic effects. We obtained a luminosity for the soft emission component of $\sim 1.2 \pm 0.4 \times 10^{40} \mathrm{erg} \mathrm{s}^{-1}$ which is consistent with that deduced from Chandra data (Colbert et al. 2005).

3. We modeled the intrinsic X-ray continuum with a power law and obtained $\Gamma=1.57_{-0.03}^{+0.06}$ for the power-law photon index.

4. We derived a column density of $8.0_{-0.5}^{+0.6} \times 10^{21} \mathrm{~cm}^{-2}$ for the line-of-sight X-ray obscuration, which corresponds to a Thomson depth of $\sim 0.006_{-0.004}^{+0.005}$ (i.e. the absorber is Compton-thin, at least in the line-of-sight). This column density is consistent with values obtained from previous BeppoSAX and $A S C A$ observations.

5. We measured $\tau_{\text {es }}=0.072 \pm 0.021$ for the optical depth of the extended, warm, electron scattering zone which is responsible for scattering $(7.2 \pm 2.1) \%$ of the intrinsic hard X-ray continuum into the line-of-sight. 
6. We detected broad and narrow Fe K line emission. When we modeled the broad and narrow lines with line emission from a relativistic disk around a Schwarzschild black hole and a Gaussian profile respectively, we found that the intensities of the broad and narrow lines were decoupled at a confidence level of greater than $3 \sigma$. We obtained an $\mathrm{EW}$ of $118_{-61}^{+32} \mathrm{eV}$, a radial line-emissivity law of $r^{-1.5(-0.8,+5.1)}$, and a lower limit of $31^{\circ}$ on the disk inclination angle from the disk-line model. The actual values are model-dependent, however.

7. The narrow Fe $\mathrm{K}$ line core $\mathrm{EW}$ was $163_{-26}^{+47} \mathrm{eV}$. The line core was unresolved by the XIS and we obtained a $90 \%$ confidence, one-parameter upper limit on the FWHM of $4850 \mathrm{~km} \mathrm{~s}^{-1}$. However, as discussed in section 2.3 the current XIS response does not model the degradation in spectral resolution. After correction for this effect, as described in section 3.2, we get an upper limit of $4630 \mathrm{~km} \mathrm{~s}^{-1}$ (or $\sigma_{N}<42 \mathrm{eV}$ ).

8. The absolute flux in the narrow line is a factor of more than 20 larger than that expected from a fully-covering spherical shell illuminated by the measured intrinsic $2-10 \mathrm{keV} \mathrm{lu}-$ minosity, having a cosmic abundance of Fe and a uniform column density equal to that measured in the line-of-sight. Therefore, roughly speaking, the product of the solid angle subtended by the line-emitting structure at the source, its angle-averaged column density, its $\mathrm{Fe}$ abundance, and the mean historical 2-10 keV luminosity averaged over timescales of the order of the structure light-crossing time, must be $>20$.

9. We detected the $\mathrm{Fe} \mathrm{K} \beta$ fluorescent line corresponding to the $\mathrm{Fe} \mathrm{K} \alpha$ line core. Taking into account residual uncertainties in the XIS energy scale calibration and conservative uncertainties on the theoretical energies of the Fe $\mathrm{K} \alpha$ and $\mathrm{Fe} K \beta$ transitions for different ionic species of $\mathrm{Fe}$, we were able to constrain the predominant ionization state of $\mathrm{Fe}$ responsible for producing the narrow $\mathrm{Fe} \mathrm{K}$ line core to be Fe I-Fe vIr. Ionization states of Fe VIII or higher are ruled out at $99 \%$ confidence. This is the most precise determination to date of the ionization state of the matter emitting the Fe $\mathrm{K}$ line core and was achieved using a method that requires no knowledge of any uncertainties in the XIS gain and any possible gravitational and Doppler shifts.

10. We were able to decouple the disk and distant-matter Fe $\mathrm{K} \alpha$ emission lines because the continuum flux was low, giving a large EW for the line core, with good signal-to-noise ratio. The large core EW also enabled the Fe K $\beta$ line to be measured with a good signalto-noise ratio, giving redundant information on the width of the distant-matter lines. This demonstrates the importance of measuring the distant-matter Fe $\mathrm{K}$ line emission parameters in order to measure those of the relativistic Fe $\mathrm{K}$ line.

11. Although the XIS data do not have the bandpass to measure the Compton-reflection continuum associated with the broad $\mathrm{Fe} \mathrm{K}$ line, we included it in all of the spectral analysis with a parameterized normalization, $R$, relative to that expected from a steady-state illumination of a disk subtending a solid angle of $2 \pi$ at the X-ray source. We obtained 
upper limits on $R$ as a function of the disk inclination angle (constrained by the relativistic Fe $\mathrm{K}$ line). We found (at $90 \%$ confidence for two parameters), $R<0.9$ for an inclination angle of $80^{\circ}$, going down to $R<0.6$ when the inclination angle is at its lower limit of $31^{\circ}$. The fact that these upper limits on $R$ are less than 1 implies that the narrow $\mathrm{Fe} K$ line core is likely to originate in Compton-thin matter.

In the future we will refine our modeling by utilizing the Suzaku HXD data when the background model has achieved the goal of $1 \%$ systematic uncertainties. The HXD data will better constrain the Compton-reflection continuum components. We will also improve model constraints by including data from XIS0 and XIS1 when the calibration has advanced further. Future observations with higher spectral resolution, such as gratings or calorimeters, will be able to resolve the narrow core of the $\mathrm{Fe} \mathrm{K} \alpha$ line and thereby better constrain the location of the line-emitter. In addition, improved throughput will enable better measurement of the profile of the broad Fe K line. Further monitoring of NGC 2992 to measure variability in the Fe K line components and in the reflection continuum as the continuum undergoes large excursions in amplitude promise to reveal important clues on the accretion process and on the structure of the central engine beyond the accretion disk.

We thank the Suzaku team and operations staff for their hard work and dedication that has made these observations of NGC 2992 possible and that has facilitated the calibration and analysis of the data. We also thank T. Kallman, T. Dotani, M. Bautz, K. Koyama, and H. Kunieda for helpful discussions and comments. TY, KM, AP, and JR acknowledge the support of NASA grant NNG04GB78A, a cooperative agreement between the Johns Hopkins University and the NASA/Goddard Space Flight Center. This research made use of the HEASARC online data archive services, supported by NASA/GSFC. This research has made use of the NASA/IPAC Extragalactic Database (NED) which is operated by the Jet Propulsion Laboratory, California Institute of Technology, under contract with NASA. 


\section{References}

Anders, E., \& Grevesse, N. 1989, Geochimica et Cosmochimica Acta 53, 197

Arnaud, K. A. 1996, in Astronomical Data Analysis Software and Systems V, ed. Jacoby, G., \& Barnes,

J. (Astronomical Society of the Pacific), Conference Series, Vol. 101, p. 17

Balucinska-Church, M., \& McCammon, D. 1992, ApJ, 400, 699

Bambynek, W., Crasemann, B., Fink, R. W., Freund, H.-U., Mark., H., Swift, C. D., Price, R. E.,

Rao, P. V. 1972, Rev. Mod. Phys., 44, 716

Bautista, M. A., Mendoza, C., Kallman, T. R., \& Palmeri, P. 2003, A\&A, 403, 339

Bearden, J. A. 1967, Rev. Mod. Phys., 39, 78

Bianchi, S., Guainazzi, M., \& Chiaberge, M. 2006, A\&A, 448, 499

Bianchi, S., Matt, G., Nicastro, F., Porquet, D., \& Dubau, J. 2005, MNRAS, 357, 599

Colbert, E. J. M., Strickland, D. K., Veilleux, S., Weaver, K. A. 2005, ApJ, 628, 113

Dickey, J. M., \& Lockman, F. J. 1990, ARA\&A, 28, 215

Dovčiak, M., Karas, V., \& Yaqoob, T. 2004, ApJS, 153, 205

Elvis, M., Fassnacht, C., Wilson, A. S., \& Briel, U. 1990, ApJ, 361, 459

Fabian, A.C., \& \& Miniutti, G. 2005, in proc. "Kerr Spacetime: Rotating Black Holes in General

Relativity" eds. D. L. Wiltshire, M. Visser \& S. M. Scott, (Cambridge Univ. Press), astro$\mathrm{ph} / 0507409$

Fabian, A.C., Rees, M.J., Stella, L., \& White, N.E. 1989, MNRAS, 238, 729

Fujimoto, R., et al. 2006, PASJ, in press

George, I. M., et al. 2002, ApJ, 571, 265

George, I. M., \& Fabian, A. C. 1991, MNRAS, 249, 352

Ghisellini, G., Haardt, F., \& Matt, G. 1994, MNRAS, 267, 743

Gilli, R., Maiolino, R., Marconi, A., Risaliti, G., Dadina, M., Weaver, K. A., \& Colbert, E. J. M. 2000, A\&A, 355, 485

Guainazzi, M., Fiore, F., Matt, G., \& Perola, G. C. 2001, MNRAS, 327, 323

Iwasawa, K., Fabian, A. C., Young, A. J., Inoue, H., \& Matsumoto, C. 1999, MNRAS, 306, L19

Iwasawa, K., Miniutti, G., \& Fabian, A. C. 2004, MNRAS, 355, 1073

Iwasawa, K., Wilson, A. S., Fabian, A. C., \& Young, A. J. 2003, MNRAS, 345, 369

Jahoda, K., et al. 2006, ApJS, 163, 401

Jiang, P., Wang, J. X., \& Wang, T. G. 2006, ApJ, 644, 725

Jiménez-Bailón, E., Piconcelli, E., Guainazzi, M., Schartel, N., Rodrguez-Pascual, P. M., Santos-Lleó, M. 2005, A\&A, 435, 449

Kallman, T. R., Palmeri, P., Bautista, M. A., Mendoza, C., \& Krolik, J. H. 2004, ApJ, 155, 675

Keel, W. C. 1996, ApJS, 106, 27

Koyama, K. et al. 2006, PASJ, in press

Krolik, J. H., \& Kallman, T. 1987, ApJ, 320, L5

Levenson, N. A., Heckman, T. M., Krolik, J. H., Weaver, K. A., \& Zycki, P. T. 2006, ApJ, in press, (astro-ph/0605438)

Magdziarz, P., \& Zdziarski, A.A. 1995, MNRAS, 273, 837 
Makishima, K. 1986, in The Physics of Accretion onto Compact Objects, ed. Mason, K. O., Watson, M. G., White, N. E. (Springer-Verlag), 266, p. 249

Matt, G., Bianchi, S., Guainazzi, M., \& Molendi, S. 2004, A\&A, 414, 155

Matt, G., Fabian, A. C., Guainazzi, M., Iwasawa, K., Bassani, L., \& Malaguti, G. 2000, MNRAS, 318, 173

Mendoza, C., Kallman, T. R., Bautista, M. A., \& Palmeri, P. 2004, A\&A, 414, 377

Mitsuda, K, et al. 2006, PASJ, in press

Molendi, S., Bianchi, S., \& Matt, G. 2003, MNRAS, 343, L1

Morrison, R., \& McCammon, D. 1983, ApJ, 270, 119

Morse, J. A., Wilson, A. S., Elvis, M., \& Weaver, K. A. 1995, ApJ, 439, 121

Nandra, K., George, I. M., Mushotzky, R. F., Turner, T. J., \& Yaqoob, T. 1997a, ApJ, 477, 602

Nandra, K., George, I. M., Mushotzky, R. F., Turner, T. J., \& Yaqoob, T. 1997b, ApJ, 488, L91

Netzer, H. 1990, in Active Galactic Nuclei, ed. R. D. Blandford, H. Netzer, \& L. Woltjer (Berlin:

Springer), 137

Ogle, P. M., Marshall, H. L., Lee, J. C., \& Canizares, C. R. 2000, ApJ, 545, L81

Page, K. L., O’Brien, P. T., Reeves, J. N., \& Turner, M. J. L.-T. 2004, MNRAS, 347, 316

Palmeri, P., Mendoza, C., Kallman, T. R., \& Bautista, M. A. 2003, A\&A, 403, 1175

Piccinotti, G., Mushotzky, R. F., Boldt, E. A., Holt, S. S., Marshall, F. E., Serlemitsos, P. J., \&

Shafer, R. A. 1982, ApJ, 253, 485

Pike, C. D., et al. 1996, ApJ, 464, 487

Ptak, A., Serlemitsos, P., Yaqoob, T., Mushotzky, R., \& Tsuru, T. 1997, AJ, 113, 1286

Reeves, J. N. 2003, in Active Galactic Nuclei: from Central Engine to Host Galaxy, ed. S. Collin, F.

Combes and I. Shlosman (Astronomical Society of the Pacific), Conference Series, Vol. 290, p. 35

Rix, H.-W., Carleton, N. P., Rieke, G., \& Reike, M. 1990, ApJ, 363, 480

Reynolds, C. S. 1997, MNRAS, 286, 513

Reynolds, C. S., \& Nowak, M. A. 2003, Phys. Rep., 377; 389

Risaliti, G. 2002, A\&A, 386, 379

Sambruna, R. M., et al. 2001, ApJ, 546, L13

Serlemitsos, P. J., et al. 2006, PASJ, in press

Sulentic, J. W., Marziani, P., Zwitter, T., Calvani, M., \& Dultzin-Hacyan, D. 1998, ApJ, 501, 54

Takahashi, T., et al. 2006, PASJ, in press

Tanaka, Y., et al. 1995, Nat, 375, 659

Turner, T. J., et al. 2002, ApJ, 574, L123

Turner, T. J., George, I. M., Nandra, K., \& Mushotzky, R. F. 1998, ApJ, 493, 91

Turner, T. J., Miller, I., George, I. M., \& Reeves, J. N. 2006, A\&A, 445, 59

Veilleux, S., Goodrich, R. W., \& Hill, G. J. 1997, ApJ, 477, 631

Weaver, K. A., Gelbord, J., \& Yaqoob, T. 2001, ApJ, 550, 261

Weaver, K. A., Nousek, J., Yaqoob, T., Mushotzky, R. F., Makino, F., \& Otani, C. 1996, ApJ, 458, 160

Weaver, K. A., \& Reynolds, C. S. 1998, ApJ, 503, L39

Woo, J.-H., \& Urry, C. M. 2002, ApJ, 579, 530 
Yaqoob, T., George, I. M., Nandra, K., Turner, T. J., Serlemitsos, P. J., \& Mushotzky, R. F. 2001, ApJ, 546, 759

Yaqoob, T., McKernan, B., Done, C., Serlemitsos, P. J., \& Weaver, K. A. 1993, ApJ, 416, L5

Yaqoob, T., \& Padmanabhan, U. 2004, ApJ, 604, 63

Yaqoob, T., Padmanabhan, U., Dotani, T., \& Nandra, K. 2002, ApJ, 569, 487

Yaqoob, T., Reeves, J. N., Markowitz, A., Serlemitsos, P. J., \& Padmanabhan, U. 2005, ApJ, 627, 156.

Young, A. J., Wilson, A. S., \& Shopbell, P. L. 2001, ApJ, 556, 6

Zhou, X.-L., \& Wang, J.-M. 2005, ApJ, 618, L83 
Table 1. NGC 2992 Suzaku Observation Log

$\begin{array}{ccccccc}\text { Obs } & \text { Start }^{a} & & \text { End }^{a} & & \text { Count Rate }^{b} & \text { Exposure } \\ & (\mathrm{UT}) & & (\mathrm{UT}) & & & \\ & & & & & & \\ & & & & & & \\ & & & & & & \\ 1 & 6 / 11 / 2005 & 14: 16: 51 & 7 / 11 / 2005 & 14: 01: 50 & 0.3888 \pm 0.0027 & 34664.5 \\ 2 & 19 / 11 / 2005 & 21: 42: 59 & 20 / 11 / 2005 & 23: 24: 45 & 0.5096 \pm 0.0032 & 31641.5 \\ 3 & 13 / 12 / 2005 & 10: 15: 37 & 14 / 12 / 2005 & 12: 08: 38 & 0.4408 \pm 0.0026 & 41681.0\end{array}$

a Start and end times correspond to the time tags of the first and last photons respectively in the cleaned and filtered events files combined from XIS2 and XIS3. ${ }^{b}$ Mean count rates and exposure times are for XIS2 and XIS3 per XIS, in the $0.5-10 \mathrm{keV}$ band. 


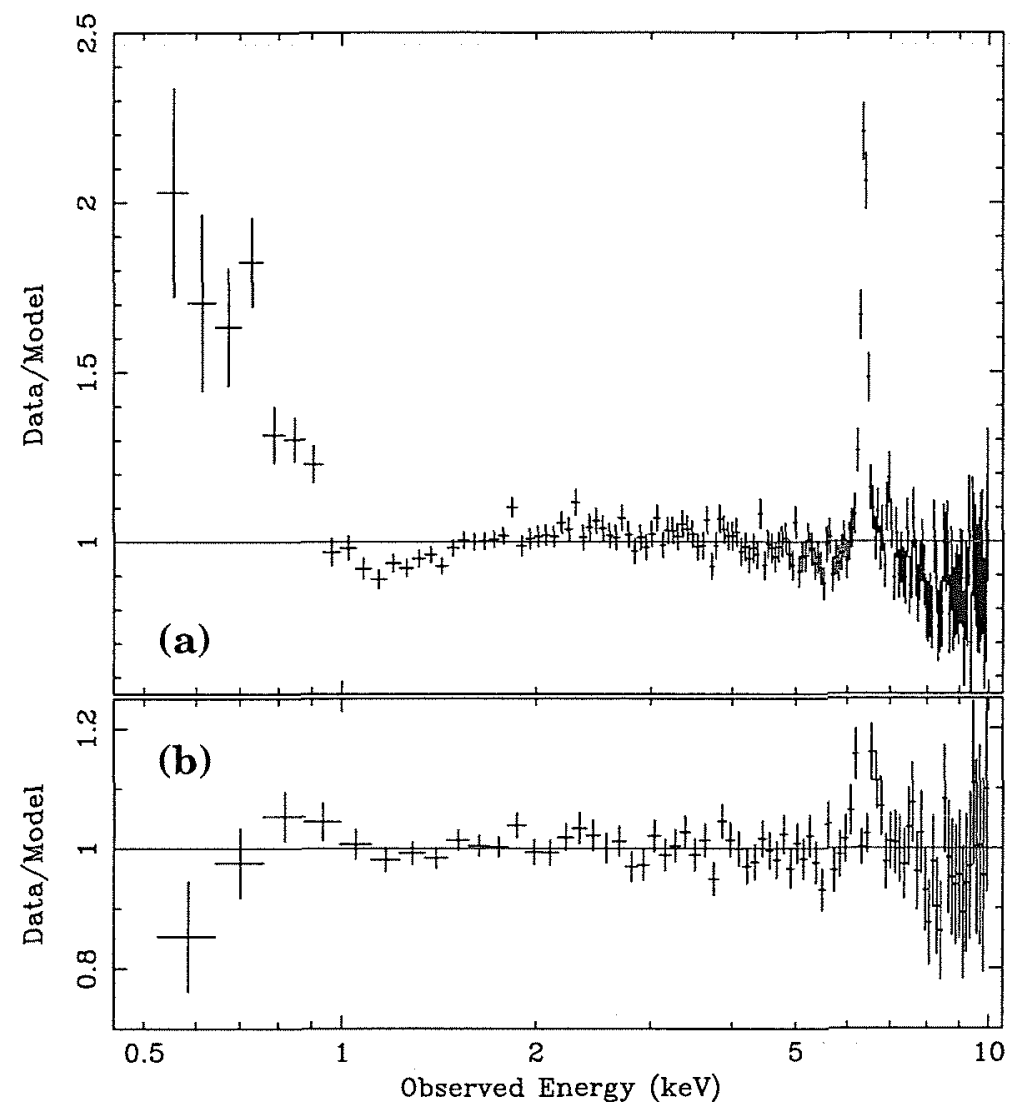

Fig. 1. (a) Ratio of the XIS2+XIS3 spectrum (integrated over observations 1 to 3 ) to a model consisting of Galactic absorption, an intrinsic power law continuum, and a uniform intrinsic absorber. (b) As (a), but now the model has additional components corresponding to an unabsorbed power-law component (for example due to scattering), and narrow (unresolved) Gaussian Fe K $\alpha$ and Fe K $\beta$ emission-line components. See section 3.1 for details. 

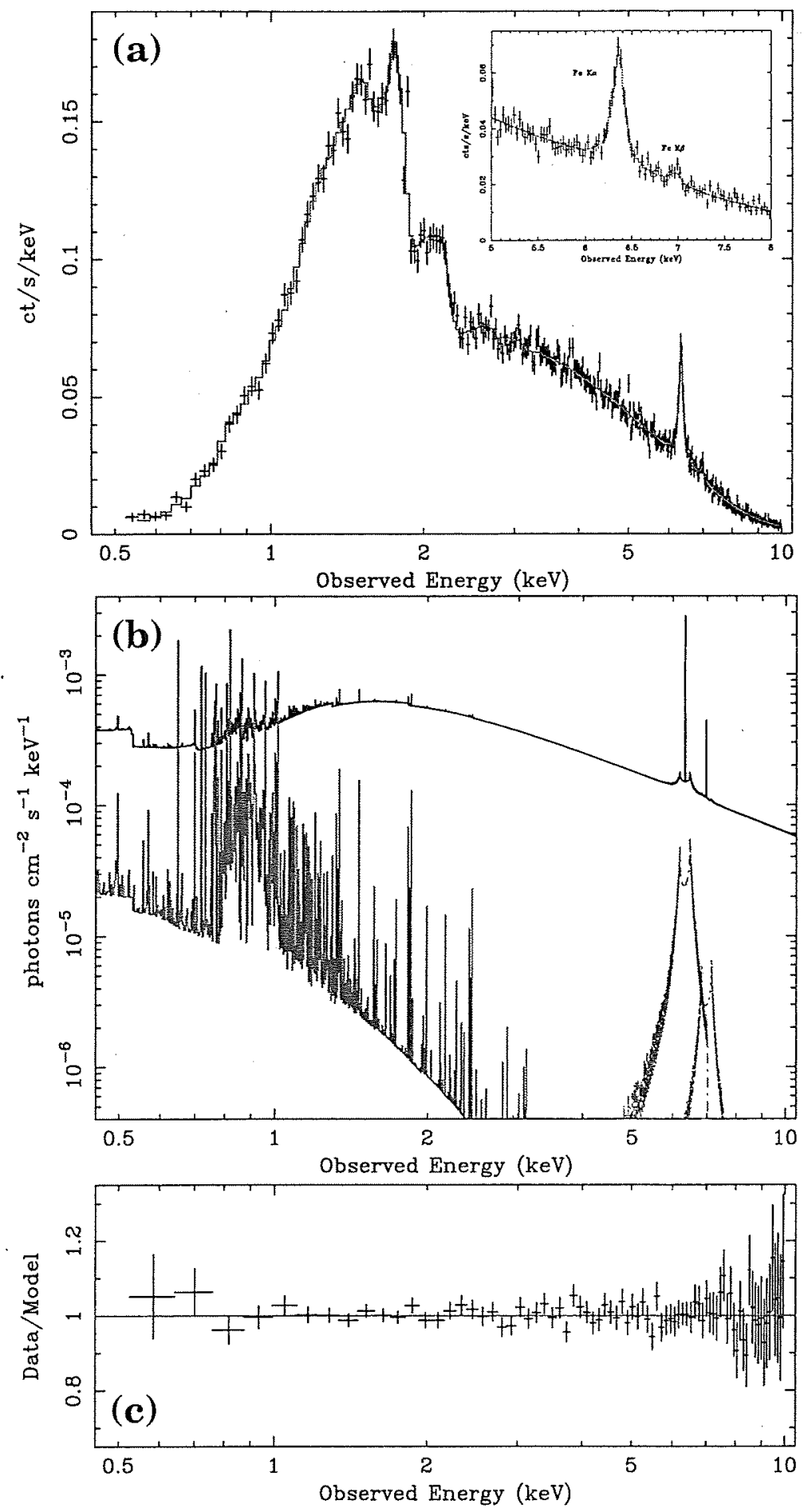

Fig. 2. (a) The XIS2+XIS3 counts spectrum (integrated over observations 1 to 3 ) overlaid with the best-fitting baseline model (solid line). The inset shows a close-up of the region containing the Fe K $\alpha$ and Fe K $\beta$ emission lines. The C/O XIS contamination is included in the model. (b) The best-fitting baseline model (see section 3.1 and table 2). The soft X-ray optically-thin thermal continuum and the broad Fe $\mathrm{K} \alpha$ and Fe $\mathrm{K} \beta$ disk-line components are also shown separately. The $\mathrm{C} / \mathrm{O}$ XIS contamination model has been removed in the plot but Galactic absorption is included. (c) The ratio of the data in (a) to the best-fitting baseline model. 

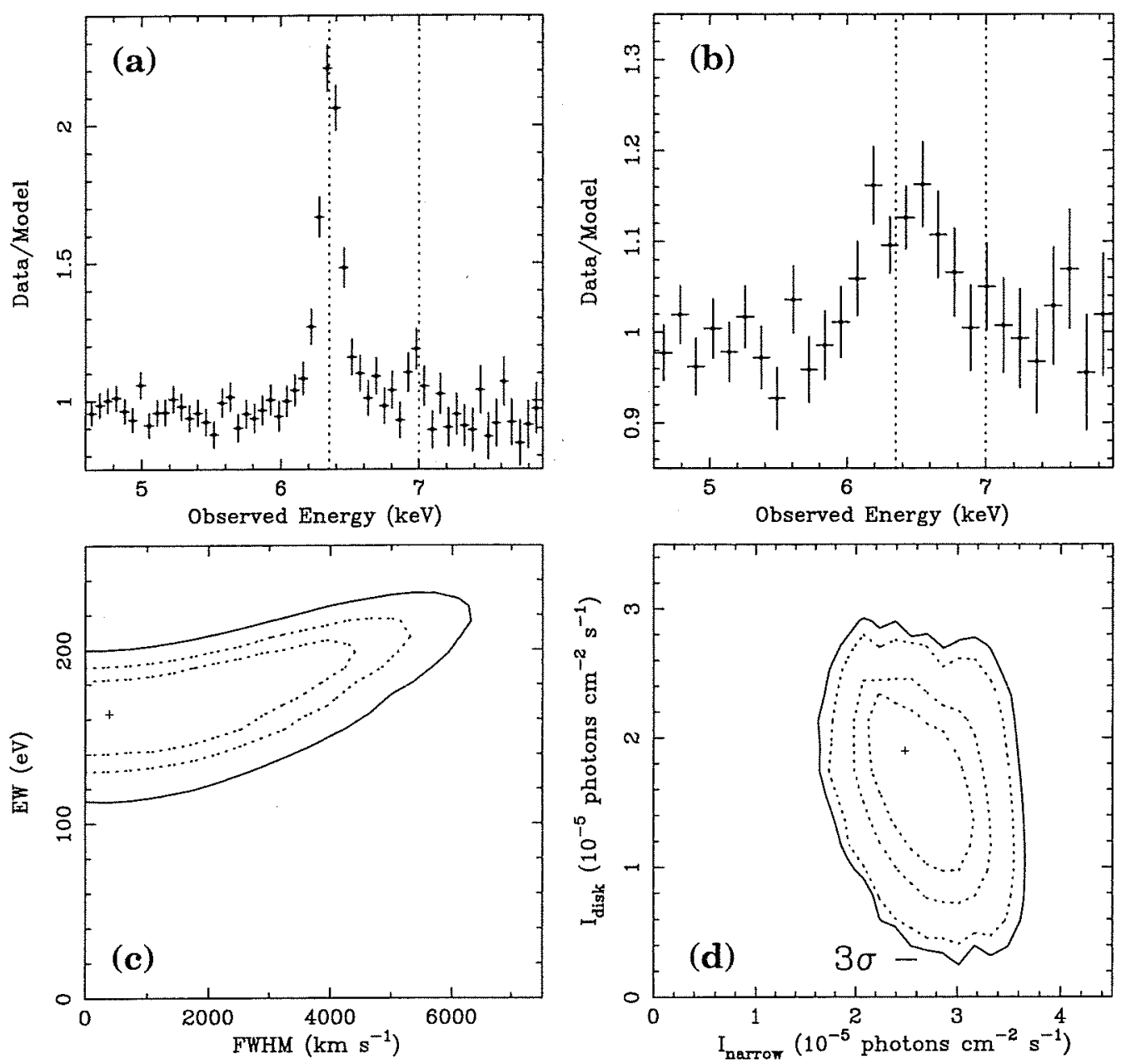

Fig. 3. (a) A close-up of the XIS spectral region in figure 1(a) containing the Fe $\mathrm{K} \alpha$ and $\mathrm{Fe} \mathrm{K} \beta$ lines. The vertical dotted lines correspond to the observed-frame energies of $\mathrm{Fe} \mathrm{I} \mathrm{K} \alpha$ and $\mathrm{K} \beta$ at $6.400 \mathrm{keV}$ and $7.056 \mathrm{keV}$ in the rest-frame respectively. All of the spectral analysis in the present paper refers to a mean spectrum constructed from XIS2+XIS3, integrated over observations I to 3. (b) The ratio of the XIS spectral data to the baseline model re-fitted after removing the relativistic $\mathrm{Fe} \mathrm{K} \alpha$ and $\mathrm{Fe} \mathrm{K} \beta$ disk-line components, whilst freezing the optically-thin thermal continuum, narrow Fe K line, and Compton-reflection continuum parameters at their best-fitting values (see section 3.2 for details). (c) The $68 \%, 90 \%, 99 \%$ confidence contours of the equivalent width (EW) versus the intrinsic width (FWHM) of the narrow, distant-matter Fe $\mathrm{K} \alpha$ line based on the best-fitting baseline model (section 3.1). The contours have not been corrected for the degradation in XIS spectral resolution (see section 3.2). (d) The $68 \%, 90 \%, 99 \%$, and $3 \sigma$ confidence contours of the Fe $\mathrm{K} \alpha$ relativistic disk-line intensity ( $\left.I_{\text {disk }}\right)$ versus the intensity of the narrow, distant-matter Fe K $\alpha$ line $\left(I_{\text {narrow }} \equiv I_{N}\right.$ ) based on the best-fitting baseline model (section 3.1). It can be seen that even at a confidence level of $3 \sigma$, the disk and distant-matter $\mathrm{Fe} \mathrm{K} \alpha$ lines are decoupled in the sense that the intensity of either of the two line components is non-zero everywhere on the $3 \sigma$ contour. 

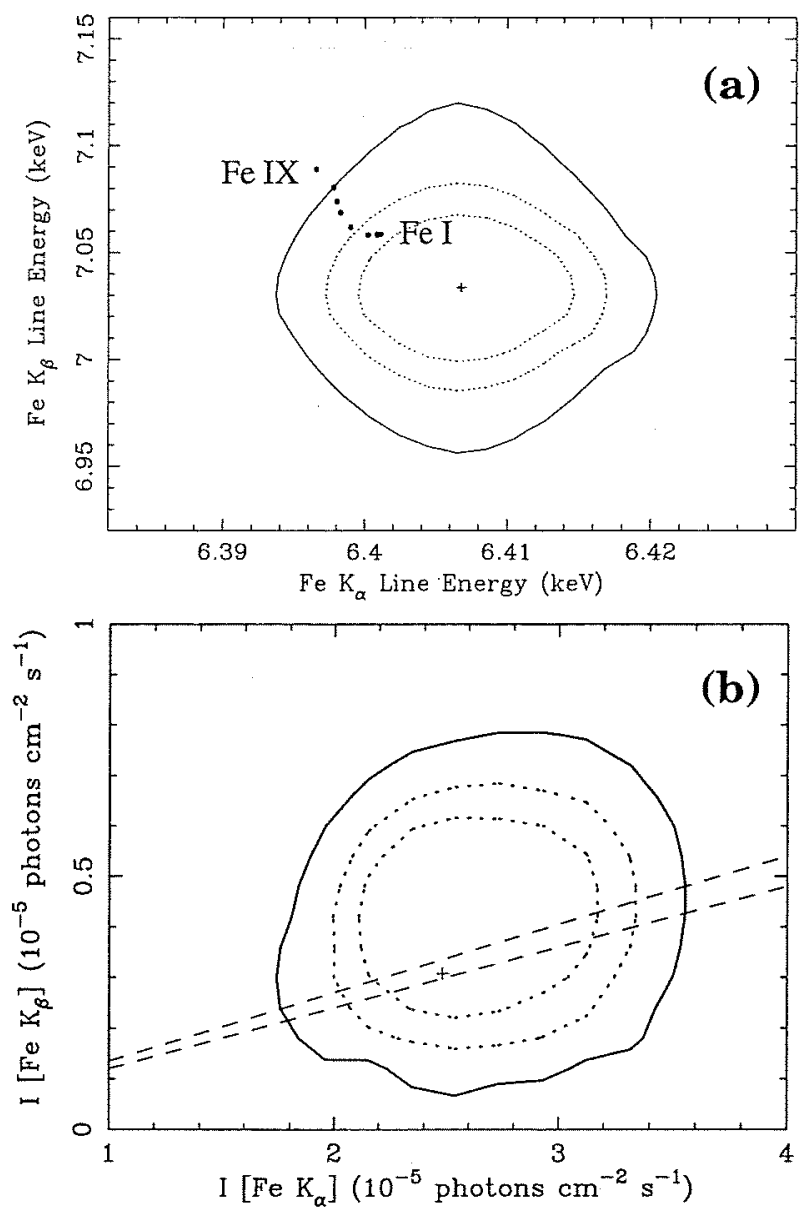

Fig. 4. (a) The $68 \%, 90 \%$, and $99 \%$ confidence contours of $E_{\mathrm{Fe} \beta}$ versus $E_{\mathrm{Fe} \alpha \alpha}$ (in the NGC 2992 rest-frame), measured from the mean XIS spectrum (see section 4.4). The filled circles are theoretical rest-frame energies of the $\mathrm{Fe} \mathrm{K} \beta$ and $\mathrm{Fe} \mathrm{K} \alpha$ emission lines from Palmeri et al. (2003). (b) The 68\%, 90\%, and $99 \%$ confidence contours of $I_{\mathrm{Fe} \mathrm{K} \beta}$ versus $I_{\mathrm{Fe} \mathrm{K} \alpha}$ (in the NGC 2992 rest-frame), measured from the mean XIS spectrum (see section 5). The lower and upper solid lines correspond to $\mathrm{Fe} \mathrm{K} \beta / \mathrm{Fe} \mathrm{K} \alpha$ branching ratios of 0.12 and 0.135 respectively (see section 5 ). 


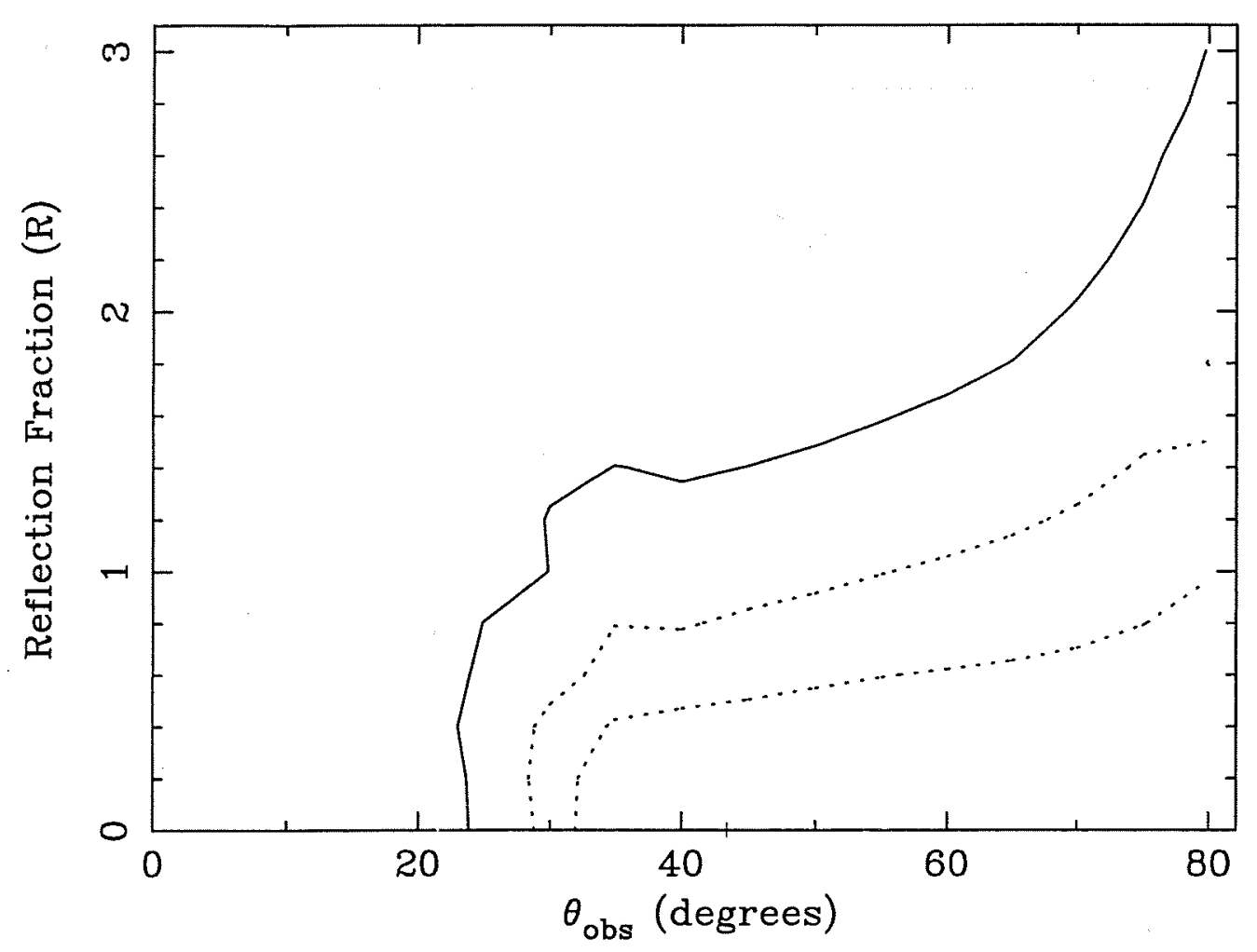

Fig. 5. The $68 \%, 90 \%, 99 \%$ confidence contours of the relative Compton-reflection fraction $(R)$ versus the inclination angle $\left(\theta_{\mathrm{obs}}\right)$ of the disk normal to the observer's line-of-sight, based on the best-fitting baseline model (section 3.1). A value of $R=1$ corresponds to a Compton-reflection continuum normalization expected from a disk subtending a solid angle of $2 \pi$ at a central non-varying X-ray source. The inclination angle parameters of the disk for the Compton-reflection continuum and relativistic disk Fe $\mathrm{K} \alpha$ line model components were tied together. See section 4.5 for details. 


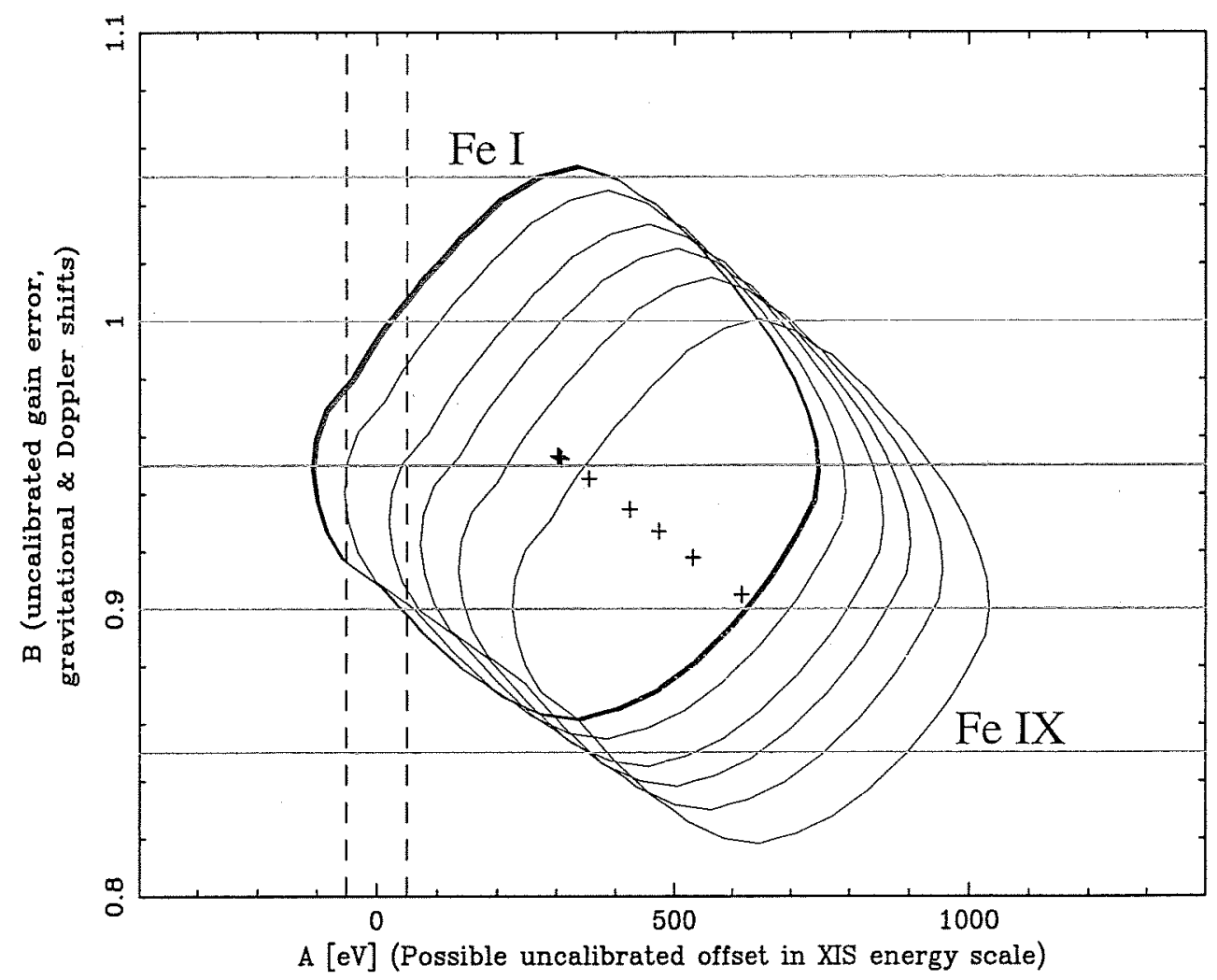

Fig. 6. The $99 \%$ confidence contours of $B$ versus $A$ calculated from the $99 \%$ contour of $E_{\mathrm{Fe}} \mathrm{K} \beta$ versus $E_{\mathrm{Fe} \mathrm{K} \alpha}$ in figure 4(a). (which was derived directly from the XIS spectrum of NGC 2992), for Fe I to Fe IX. The method of transformation of the $E_{\mathrm{Fe} K} \beta$ versus $E_{\mathrm{Fe} \mathrm{K} \alpha}$ contour is described in section 5 . The Fe $\mathrm{K} \alpha$ and $\mathrm{Fe} \mathrm{K} \beta$ fluorescent line energies were calculated using atomic data in Palmeri et al. (2003) and are also shown as filled circles in figure 4(a). A represents residual uncertainties in the offset of the absolute energy scale which is believed to be on the order of $\sim 10 \mathrm{eV}$, but the dashed vertical lines are placed very conservatively at $\pm 50 \mathrm{eV} . B$ represents the product of any gravitational shift, Doppler shift, or residual uncorrected gain factors. Contours of $B$ versus $A$ that do not have any portion lying inside $A= \pm 50 \mathrm{eV}$ are ruled out at $99 \%$ confidence. 
Table 2. XIS Spectral Fitting Results for NGC 2992

$\chi^{2} /$ degrees of freedom $\quad 334.4 / 309$

$\begin{array}{lr}k T(\mathrm{keV}) & 0.658_{-0.061}^{+0.088} \\ L_{\mathrm{APEC}}\left(10^{40} \mathrm{erg} \mathrm{s}^{-1}\right) & 1.18_{-0.45}^{+0.36}\end{array}$

$\Gamma$

$N_{H}\left(10^{21} \mathrm{~cm}^{-2}\right)$

$f_{\mathrm{s}}$ (scattered fraction)

$\theta_{\text {obs }}$ (degrees)

$q$

$I_{\text {disk }}[$ Fe K $\alpha]\left(10^{-5}\right.$ photons $\left.\mathrm{cm}^{-2} \mathrm{~s}^{-1}\right)$

$\mathrm{EW}_{\text {disk }}[\mathrm{Fe} \mathrm{K} \alpha](\mathrm{eV})$

$E_{N}[\mathrm{Fe} \mathrm{K} \alpha](\mathrm{keV})$

$\sigma_{N}(\mathrm{keV})^{a}$

FWHM $\left(\mathrm{km} \mathrm{s}^{-1}\right)^{a}$

$I_{\mathrm{N}}[\mathrm{Fe} \mathrm{K \alpha}]\left(10^{-5}\right.$ photons $\left.\mathrm{cm}^{-2} \mathrm{~s}^{-1}\right)$

$\mathrm{EW}_{\mathrm{N}}[\mathrm{Fe} \mathrm{K \alpha}](\mathrm{eV})$

$E_{N}[\mathrm{Fe} \mathrm{K} \beta](\mathrm{keV})$

$I_{\mathrm{N}}[\mathrm{Fe} \mathrm{K} \beta]\left(10^{-5}\right.$ photons $\left.\mathrm{cm}^{-2} \mathrm{~s}^{-1}\right)$

$\mathrm{EW}_{\mathrm{N}}[\mathrm{Fe} \mathrm{K} \beta](\mathrm{eV})$

$I_{\mathrm{N}}[\mathrm{Ni} \mathrm{K \alpha}]\left(10^{-5}\right.$ photons $\left.\mathrm{cm}^{-2} \mathrm{~s}^{-1}\right)$

$\mathrm{EW}_{\mathrm{N}}[\mathrm{Ni} K \alpha](\mathrm{eV})$

Reflection fraction, ${ }^{b} R\left(\theta_{\text {obs }}=43^{\circ}\right)$

Reflection fraction, ${ }^{b} R\left(\theta_{\text {obs }}=60^{\circ}\right)$

$F_{0.5-2 \mathrm{keV}}^{c}\left(10^{-11} \mathrm{erg} \mathrm{cm}^{-2} \mathrm{~s}^{-1}\right)^{b}$

$F_{2-10 \mathrm{keV}}^{c}\left(10^{-11} \mathrm{erg} \mathrm{cm}^{-2} \mathrm{~s}^{-1}\right)^{b}$

$L_{0.5-2 \mathrm{keV}}^{d}\left(10^{42} \operatorname{erg~s}^{-1}\right)^{c}$

$L_{2-10 \mathrm{keV}}^{d}\left(10^{42} \mathrm{erg} \mathrm{s}^{-1}\right)^{c}$

$$
\begin{array}{r}
1.569_{-0.027}^{+0.056} \\
7.99_{-0.45}^{+0.56} \\
0.073_{-0.021}^{+0.021}
\end{array}
$$

$>31$

$-1.5_{-0.8}^{+5.1}$

$1.9_{-1.0}^{+0.5}$

$118_{-61}^{+32}$

$6.407_{-0.007}^{+0.007}$

$<0.042$

$<4630$

$2.49_{-0.40}^{+0.71}$

$163_{-26}^{+47}$

$7.034_{-0.038}^{+0.037}$

$0.43_{-0.23}^{+0.20}$

$27_{-14}^{+12}$

$<0.29$

$<28$

$<0.60$

$<0.78$

0.16

1.15

0.70

1.60

All values and energy ranges in this table (except $F_{0.5-2 \mathrm{keV}}$ and $F_{2-10 \mathrm{keV}}$ ) refer to the rest-frame of NGC $2992(z=0.00771)$. Statistical errors are for $90 \%$, one interesting parameter $\left(\Delta \chi^{2}=2.706\right)$ and were derived with fifteen parameters free, with $I_{\mathrm{Ni}}$ Fe K $\alpha$ fixed at 0 . Upper limits on the intensity and equivalent width (EW) of the $\mathrm{Ni} \mathrm{K} \alpha$ line were derived with sixteen parameters free. a The upper limits on the widths of the line core of $\mathrm{Fe} \mathrm{K \alpha}$ and $\mathrm{Fe} \mathrm{K} \beta$ have been corrected (as described in section 3.2) for the degradation of the XIS spectral resolution. ${ }^{b}$ Upper limits on $R$ are for $\theta_{\text {obs }}$ fixed at the best-fitting value of $43^{\circ}$ and for $\theta_{\text {obs }}=60^{\circ}$, as indicated. ${ }^{c}$ Observed-frame fluxes, corrected for the CCD contamination but not corrected for Galactic and intrinsic absorption. ${ }^{d}$ Intrinsic, rest-frame luminosities, corrected for all absorption components. 